\title{
Dynamic generation of maximally entangled photon multiplets by adiabatic passage
}

\author{
W. Lange* and H. J. Kimble \\ Norman Bridge Laboratory of Physics 12-33, California Institute of Technology, Pasadena, California 91125
}

(Received 10 August 1999; published 17 May 2000)

\begin{abstract}
The adiabatic passage scheme for quantum state synthesis, in which atomic Zeeman coherences are mapped to photon states in an optical cavity, is extended to the general case of two degenerate cavity modes with orthogonal polarization. Analytical calculations of the dressed-state structure and Monte Carlo wave-function simulations of the system dynamics show that, for a suitably chosen cavity detuning, it is possible to generate states of photon multiplets that are maximally entangled in polarization. These states display nonclassical correlations of the type described by Greenberger, Horne, and Zeilinger (GHZ). An experimental scheme to realize a GHZ measurement using coincidence detection of the photons escaping from the cavity is proposed. The correlations are found to originate in the dynamics of the adiabatic passage and persist even if cavity decay and GHZ state synthesis compete on the same time scale. Beyond entangled field states, it is also possible to generate entanglement between photons and the atom by using a different atomic transition and initial Zeeman state.
\end{abstract}

PACS number(s): 42.50.Dv, 03.65.Bz

\section{INTRODUCTION}

A quantum system consisting of several components is called entangled if its state cannot be expressed as a direct product of substates for the constituents. Such a system has no classical analog and hence shows distinct quantum mechanical features. Of particular interest are multiplets of particles in maximally entangled states, in which a measurement of the energy eigenvalue of one particle completely determines the outcome of the same measurement for all the others. An example in the case of two particles are the EPR or Bell states [1], which give the largest contradiction with the local realism assumption of Einstein, Podolsky, and Rosen [2]. Greenberger, Horne, and Zeilinger [3,4] and later Mermin [5] have shown that a much stronger and more direct test of local realism may be achieved by using three or more maximally entangled particles. Violation of local realism can then be obtained in a single set of experiments, whereas EPR correlations between two particles show up only statistically in a series of measurements.

A number of different experimental schemes have been proposed to generate a maximally entangled or GHZ state involving three or more photons or atoms [3]. Proposals for optical experiments typically use photon pairs generated in parametric down conversion to obtain GHZ correlations [6-8] or polarization correlations among photons emitted in cascaded atomic decays [4]. Other schemes suggest utilizing the QED interaction of atoms and field modes of a cavity to generate entanglement. Three or four two-level Rydberg atoms may be prepared in an entangled state through their interaction with the field in a microwave cavity [9-12]. Conversely, three microwave cavities may be entangled due to the interaction with a Rydberg atom [13]. Yet another suggestion uses a single photon in a double Mach-Zehnder interferometer to create a three-atom GHZ state [14]. There are

\footnotetext{
*Present address: Max-Planck-Institut für Quantenoptik, D-85748 Garching, Germany. Electronic address: wfl@mpq.mpg.de
}

also mixed schemes with entanglement between different types of subsystems, for example, four cavity modes and a single atom [15]. While in most proposals the entangled particles originate in a single localized source, GHZ correlations may also be produced using particles from separate sources $[16,17]$. In this way recently photon states were realized that retrodictively reproduce GHZ statistics of photocounts [18]. Another system that should be capable of producing entanglement are two-level ions in a linear quadrupole trap, subjected to appropriately timed pulses [19]. Some of these schemes allow the subsystems to be probed in spatially separated locations, which is important if the issues of nonseparability and nonlocality in quantum mechanics are to be investigated. In other cases such a separation of the subsystems is impossible in principle, thus precluding tests of the causal dependence between the measurements. In spite of the many theoretical proposals, however, to date no experimental realization of a GHZ state with three or more particles has been achieved.

Here, we present a novel approach to the generation of GHZ correlations, adapting a method from the field of quantum-state synthesis in cavity QED [20-22]. The particles we consider are photons in a single longitudinal mode of an optical resonator. One way to create "arbitrary" nonclassical states of the field in such a system is the method of adiabatic passage $[20,23,24]$. In this protocol, an atom that is prepared in a coherent superposition of Zeeman ground states sequentially traverses a resonant quantized cavity mode and a coherent-state laser field with overlapping spatial profiles. If the transit is slow enough (adiabatic), the system remains in a single quantum state and the coherence of the Zeeman sublevels is mapped to the photon distribution of the cavity mode. Therefore, the number of quantum states of the field that may be synthesized is only limited by the possible Zeeman state superpositions of the atom. Previous work $[20,23]$ shows this scheme to be robust, without the requirement for precise control of pulse area or timing.

In our scheme for the generation of a GHZ state, the cavity photons are entangled in polarization. Since in the 
previous theory of adiabatic passage [20,23] only a single polarization was considered (which was taken to be $\sigma_{-}$, implying an infinitely detuned $\sigma_{+}$-polarized mode), we start in Sec. II by extending the method to the case of two degenerate orthogonal polarizations for the cavity mode. The model allows us to find a straightforward procedure for the synthesis of a GHZ state of polarization entangled photons and to calculate the efficiency of its generation. Throughout the paper we utilize parameters already realized in present day cavity QED experiments. Note that from an experimental point of view, our results are relevant even for the generation of Fock states of a single polarization, since in realistic Fabry-Pérot resonators, modes with both transverse polarizations are always present. Although in practice small biases towards one polarization may occur (e.g., due to birefringence of the mirrors), our model assumes that the frequency splitting of the polarization modes of the cavity is negligible (degenerate case). However, in Sec. IV we present numerical calculations for finite mode splitting (in the circular polarization basis) to derive a quantitative criterion for the validity of the degenerate model. These results also show how large a mode splitting is necessary for a single transverse mode description of the system to be applicable.

In the single polarization case [20,23], the adiabatic dynamics evolves in a one-dimensional space along the socalled dark state, a superposition of ground states which is decoupled from excited atomic levels. Each atomic ground state within the manifold of Zeeman substates is correlated with one (and only one) photon number of the cavity field, as any given Zeeman state is reached by a unique sequence of photon absorption and emission cycles. Thus a shift of the atomic population is always accompanied by a corresponding shift of the photon distribution. By contrast, with both (degenerate) polarizations of the cavity field taken into account, the system behavior is considerably more complex. Population may be shifted to higher or lower magnetic sublevels, depending on the polarization of the photons involved. As a consequence there is no intrinsic limit to the number of photons that may be generated by adiabatic passage. The resulting final states of the cavity field are no longer expected to be pure Fock states, but a complex superposition of number states for both polarizations, even when the initial atomic state is a single Zeeman substate. This is shown in Sec. III by analyzing the structure of the relevant eigenstates.

After characterizing the dynamics of state synthesis by adiabatic passage with two polarizations, we proceed by proposing a new strategy to arrive at pure Fock states of the cavity field by making use of a suitable detuning of the cavity field. The feasibility of the method is demonstrated in Sec. IV by a quantum Monte Carlo simulation of the system dynamics. An important result is that there is no restriction on our method with respect to polarization: the scheme is capable of generating number states of either cavity polarization or even arbitrary superpositions of both. The last fact opens the door for the generation of a GHZ state of polarization entangled photons starting from a suitable superposition of Zeeman ground states. Details of this new protocol are explained in Sec. V.

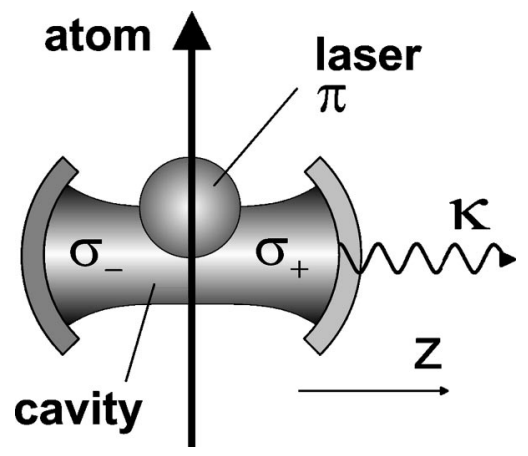

FIG. 1. Setup of the two-polarization adiabatic passage scheme. With spatial quantization along the $z$ axis, the cavity supports a $\sigma^{+}$and a degenerate $\sigma^{-}$-polarized mode. The atomic beam successively traverses the cavity waist and the $\pi$-polarized pump laser beam, which is oriented perpendicular to the direction of the atoms and the cavity axis.

In a realistic experiment, the synthesis of a GHZ state in a cavity will certainly be affected by energy dissipation. In Sec. VI we incorporate this fact into the model and utilize it to detect GHZ correlations by coincidence measurements of the photons escaping from a leaky cavity. Somewhat surprisingly, we find that even when decay sets in before the generation of the GHZ state is finished (i.e., when the cavity decay and adiabatic passage times are comparable), correlations between the photons persist. Obviously, these correlations originate in the dynamics of the adiabatic process rather than in the properties of a stationary final state of the system.

A variation of the method that creates entanglement among photons and atoms is presented in Sec. VII. Here, the initial state for the adiabatic passage is a single Zeeman ground state, which may be easily prepared by optical pumping with $\pi$-polarized light. GHZ correlations are detected by a coincidence measurement of the photons emitted from the cavity and information on the atomic state obtained from a "Stern-Gerlach" device. Our results are summarized in Sec. VIII.

\section{MODEL OF THE ATOM-CAVITY SYSTEM}

The experimental configuration we consider is similar to the one presented in [20] and is depicted in Fig. 1. A moving atom (transition frequency $\omega_{a}$ ) first encounters the waist of a quantized cavity field and subsequently a pump-laser field injected perpendicular to both cavity axis and the atom's direction of propagation. Cavity and pump fields overlap partially, so that there is a region where in the rest frame of the atom the pump field increases as the cavity field decreases. The cavity field is described by two transverse-polarization states of a single longitudinal mode. With the cavity axis as the direction for spatial quantization, the polarizations are taken to be $\sigma^{+}$and $\sigma^{-}$and the resonance frequencies of the corresponding modes denoted as $\omega_{c \pm}$. The ability to accomodate arbitrary polarization of the cavity field distinguishes the model presented here from that of Refs. [20,23]. It not only allows us to investigate polarization entangled states of the cavity field, such as the maximally entangled GHZ states, but also leads to an improved scheme for the generation of single-polarization Fock states in a realistic experimental situation. 


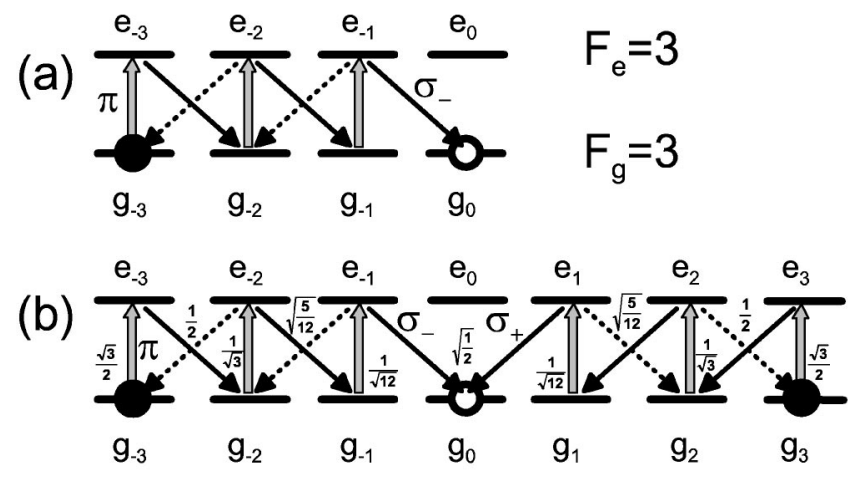

FIG. 2. Scheme of the $\left(F_{g}=3\right)$ and $\left(F_{e}=3\right)$ hyperfine levels, showing the transitions relevant for adiabatic passage starting from two different initial preparations of Zeeman sublevels (indicated by the solid circles): (a) $m_{g}=-3$; (b) superposition of $m_{g}=-3$ and $m_{g}=+3$. The wide arrows symbolize the $\pi$-polarized (classical) pump transitions. The predominant cavity-induced transition from a given excited state $\mathrm{e}_{m}$ [larger Clebsch-Gordan coefficient as indicated by the numbers in diagram (b)] is drawn as a solid, the weaker emission into the orthogonal polarization mode as a dotted arrow. Note that at the end of the passage the atom is always found in the $m_{g}=0$ level (open circle), even in the presence of two cavity polarizations.

We assume here that the atomic levels involved are the Zeeman substates of ground and excited levels $F_{g}$ and $F_{e}$, although more general configurations can also be employed $[22,25,26]$. In most of the paper we concentrate on the case $F_{g}=F_{e}$, where the transition $\left(m_{g}=0\right) \rightarrow\left(m_{e}=0\right)$ is electric dipole forbidden, so that a $\pi$-polarized pump beam does not couple to atoms in the $m_{g}=0$ state of the lower Zeeman manifold. Therefore, if by a suitable interaction with pump field and cavity mode, atomic population is pushed towards levels with decreasing $\left|m_{g}\right|$, the process will terminate once the level $\left(m_{g}=0\right)$ is reached, independent of the initial atomic distribution. Note that the coupling coefficients among the various Zeeman states for the $F_{g} \leftrightarrow F_{e}$ transition are symmetric under a change of sign of the magnetic quantum number, which is important for the generation of states involving photons of different polarization.

The maximum number of photons that can be generated with the scheme is achieved when the atom is prepared in the outer Zeeman ground states $\left(m_{g}= \pm F_{g}\right)$ or a coherent superposition of both. As shown below, ideal adiabatic passage in this case will generate $F_{g}$ (entangled) photons distributed among both cavity modes. With cesium (nuclear spin $I$ $=7 / 2, F_{g}=3$ or 4 ) as an example, states with three or four entangled photons are therefore possible. Figure 2 shows the relevant pump and cavity-induced transitions in the case $F_{g}$ $=F_{e}=3$ for two possible initial preparations of the Zeeman sublevels.

In our model, the cavity field is described by annihilation and creation operators $a_{+}, a_{+}^{\dagger}$ for the $\sigma_{+}$-polarized mode and $a_{-}, a_{-}^{\dagger}$ for $\sigma_{-}$polarization, while the pump field is treated classically (i.e., as a coherent state in a regime of weak coupling). The atomic degrees of freedom are described by operators $A_{\sigma}$, which are composed of a sum over spin-1/2 operators for the Zeeman levels $\left|F_{g}, m_{g}\right\rangle$ and
$\left|F_{e}, m_{e}\right\rangle$ with weights corresponding to the Clebsch-Gordan coefficients for dipole coupling with polarization $\sigma$, namely

$$
A_{\sigma}=\sum_{m_{g}, m_{e}}\left|F_{g} m_{g}\right\rangle\left\langle F_{g} m_{g} ; 1 \sigma \mid F_{e} m_{e}\right\rangle\left\langle F_{e} m_{e}\right|, \quad \sigma=0, \pm 1 .
$$

As the atom moves across the cavity, it undergoes a timedependent interaction with the fields, which is expressed by a variable coupling constant $g(t)$ for the two quantized cavity modes and a Rabi frequency $\Omega(t)$ for the classical pump field. The time scale is determined by the atomic velocity. Alternative time-dependent coupling schemes for the case of a stationary atom located in the cavity field $[22,25,26]$ may be treated with the same formalism.

With the above definitions, the Hamiltonian for the atom and the two fields in a frame rotating at the frequency $\omega_{l}$ of the classical field $\Omega(t)$ is given by

$$
\begin{aligned}
H_{\mathrm{int}}(t)= & \hbar \delta_{+} a_{+}^{\dagger} a_{+}+\hbar \delta_{-} a_{-}^{\dagger} a_{-}-i \hbar g(t)\left(a_{+}^{\dagger} A_{+1}-A_{+1}^{\dagger} a_{+}\right) \\
& -i \hbar g(t)\left(a_{-}^{\dagger} A_{-1}-A_{-1}^{\dagger} a_{-}\right)+i \hbar \Omega(t)\left(A_{0}-A_{0}^{\dagger}\right) .
\end{aligned}
$$

Here, $\delta_{ \pm}=\omega_{c \pm}-\omega_{l}$ is the detuning between the cavity frequency $\omega_{c \pm}$ for the $\sigma_{ \pm}$-polarized mode and the classical field, which is assumed to be at resonance with the atomic transition $\left(\omega_{a}=\omega_{l}\right)$, throughout the present analysis.

A basis for the Hilbert space of the atom-cavity system is provided by tensor products of the atomic states and the number states for each polarization of the cavity mode. We use the notation

$$
\left|x_{m}, n_{+}, n_{-}\right\rangle=\left|x_{m}\right\rangle \otimes\left|n_{+}\right\rangle \otimes\left|n_{-}\right\rangle,
$$

with $x=e, g$ specifying excited and ground state, $m=$ $-F_{x}, \ldots, F_{x}$ the number of the magnetic sublevel, and $n_{ \pm}$ the photon number in the respective cavity mode.

In this paper the initial preparation of the system is not described explicitly. We assume that before an atom enters the interaction zone both modes of the cavity field are in the vacuum state and the atom is prepared in an arbitrary coherent superposition of Zeeman ground states. This may be achieved by radio-frequency pumping [27] or a series of optical Raman pulses [28].

In our initial discussion in the following section, atomic spontaneous emission and cavity decay will be neglected. They will be included in Secs. IV and V in a master equation approach using the quantum Monte Carlo wave-function technique.

\section{DRESSED STATES AND DARK SPACE}

Fundamental aspects of the system evolution in the twopolarization adiabatic passage scheme may be inferred from the structure of the eigenvalues and eigenvectors of $H_{\text {int }}(t)$ during the transit of a single atom. As a specific example we consider the $\left(6 S_{1 / 2}, F_{g}=3\right) \rightarrow\left(6 P_{1 / 2}, F_{e}=3\right)$ transition in cesium (cf. Fig. 2) at $852.36 \mathrm{~nm}$. For the system parameters we have chosen values reached in recent cavity QED experi- 


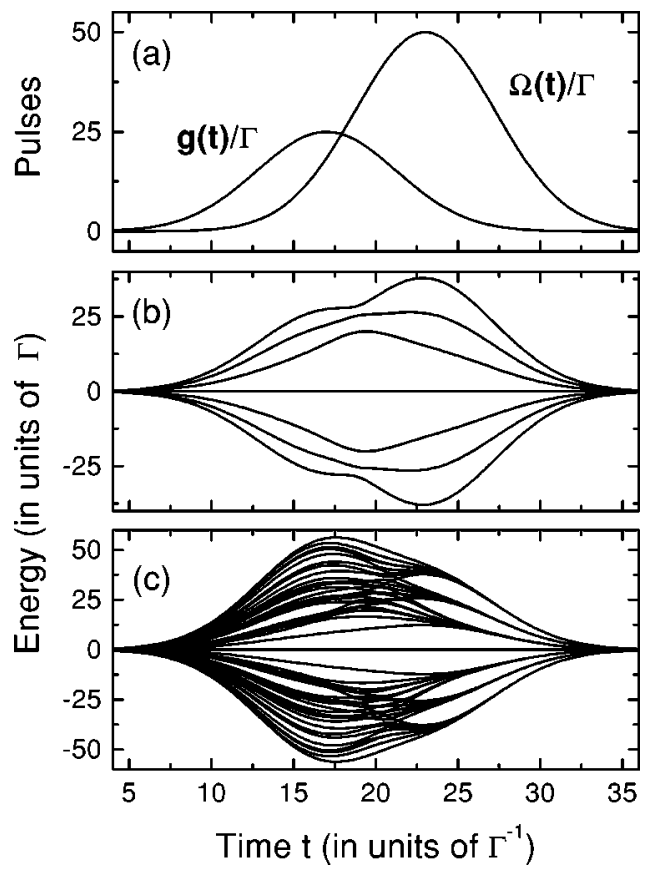

FIG. 3. (a) Time evolution of the coupling $g(t)$ and Rabi frequency $\Omega(t)$ of the pump field, which are assumed to be Gaussian pulses with FWHM $10 \Gamma^{-1}$; they are centered at $t=17 \Gamma^{-1}$ and $t$ $=23 \Gamma^{-1}$, respectively, and have amplitudes $g_{0}=25 \Gamma$ and $\Omega_{0}$ $=50 \Gamma$; (b) energy eigenvalues of $H_{\text {int }}$ in the single-polarization case; (c) energy eigenvalues for two polarizations. Atom, cavity modes, and pump field are at resonance $\left(\omega_{a}=\omega_{c+}=\omega_{c-}=\omega_{l}\right)$.

ments with high finesse optical resonators [29-33]. In particular, a peak atom-cavity coupling $g_{0}=25 \Gamma$ on the resonator axis was used with $\Gamma / 2 \pi=5 \mathrm{MHz}$ being the spontaneous emission rate for the cesium D2 line (i.e., $\Gamma^{-1}$ is the population lifetime). Both cavity modes and the pump beam are assumed to have a Gaussian transverse shape with a full width at half maximum (FWHM) $w=10 \Gamma^{-1}$, but the pump beam has its center displaced along the atomic beam by an amount of $0.6 w$ relative to the cavity mode [Fig. 3(a)]. The maximum Rabi frequency $\Omega_{0}$ of the pump field is chosen as twice the coupling constant $g_{0}$ (i.e., $\Omega_{0}=50 \Gamma$ for the above specified coupling). These values were found to yield optimum results in the single polarization case $[20,23]$ as well as in the present work. Small variations in the shape and intensity of the field distributions on the order of a few percent, however, do not lead to substantially different results.

With the two cavity modes in the vacuum state and the atom prepared in the lowest Zeeman ground state $\left(m_{g}=\right.$ -3 ), the initial wave fuction in the notation of Eq. (3) is

$$
\left|\Psi_{\text {ini }}\right\rangle=\left|g_{-3}, 0,0\right\rangle \text {. }
$$

The subsequent analysis will be restricted to the manifold of states that are coupled to $\left|\Psi_{\text {ini }}\right\rangle$ by the interaction Hamiltonian $H_{\text {int }}$.

In order to compare the system behavior for the cases of one and two polarizations, we first calculate the eigenvalues of $H_{\text {int }}$ when only the $\sigma_{-}$mode interacts with the atom, corresponding to the single polarization analysis of Refs.
[20,23]. To this end the terms involving $a_{+}$-operators are omitted from the Hamiltonian (2). Figure 3(b) shows the eigenvalues obtained as a function of transit time for typical experimental parameters and a cavity detuning $\delta_{-}=0$. There are seven nondegenerate energy levels that are also referred to as dressed states since they describe the energy of atom and the surrounding fields. The dressed state employed for the adiabatic passage is the one that does not couple to excited atomic states and hence does not experience an acStark shift due to either the cavity or pump fields (dark state). Its energy eigenvalue is $E=0$ and the corresponding eigenvector in the basis (3), up to a normalization factor, is

$$
\begin{aligned}
\left|E_{0}\right\rangle= & -\sqrt{15} g^{3}(t)\left|g_{-3}, 0,0\right\rangle+\sqrt{45} g^{2}(t) \Omega(t)\left|g_{-2}, 0,1\right\rangle \\
& -\sqrt{18} g(t) \Omega^{2}(t)\left|g_{-1}, 0,2\right\rangle+\Omega^{3}(t)\left|g_{0}, 0,3\right\rangle .
\end{aligned}
$$

This state has the property that if the system is prepared in $|g-3,0,0\rangle$ in a region of $\Omega \approx 0$, then slowly increasing $\Omega(t)$ while reducing $g(t)$ to zero $(g / \Omega \approx 0)$ will adiabatically transform the initial state to $\left|g_{0}, 0,3\right\rangle$, i.e., a state with three $\sigma_{-}$photons in the cavity mode. As is apparent from Eq. (5), the creation of each photon is accompanied by an increase in the magnetic quantum number $m_{g}$ of the atom. Since for a $\pi$-polarized classical laser field no more pumping can occur once the state $m_{g}=0$ is reached, exactly three photons will be found in the cavity mode at the end of the adiabatic passage. This method has been analyzed as a scheme for the generation of Fock states and for more general superposition states of the field arising from superpositions of initial Zeeman states in the $F_{g}$ manifold [20,23].

These conclusions are no longer valid if a second, orthogonally polarized cavity mode near resonance is taken into account, as a calculation of the eigenvalues of $H_{\text {int }}$ for two cavity polarizations with equal frequencies demonstrates. According to Fig. 3(c), the resulting level structure is considerably more complex than in the single polarization case, with a large number of levels undergoing crossings and anticrossings. This is due to the fact that in contrast to the single-polarization case there is no intrinsic limit to the number of photons that may be deposited in the cavity mode, as will be explained below. The numerical simulations (Sec. IV) show that not all the levels accessible to the system contribute equally to the dynamics. For example, a cutoff of six photons in both cavity modes was found to produce sufficiently accurate results and was used in Fig. 3(c).

Another important implication of the second polarization is that the dark state at the energy $E=0$ becomes highly degenerate, the degree of degeneracy being limited only by the cutoff photon number. There is no longer a single dark state but rather a dark space in which the dynamics evolve in the adiabatic case. Consequently, no simple final state of the atom-cavity system is expected to be generated by the adiabatic passage, but rather a complex superposition state in the dark subspace.

In the single polarization case only one fundamental process is involved in the evolution of the dark state (5), in which a photon from the pump field is scattered into the $\sigma_{-}$ cavity mode, increasing the magnetic quantum number by 
(a)

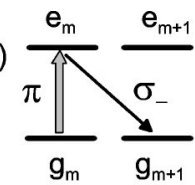

(b)

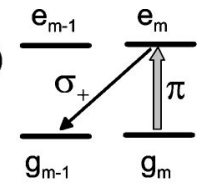

(c)

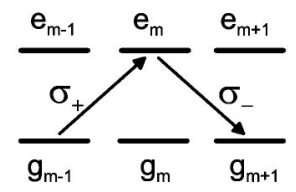

FIG. 4. Fundamental processes of the dynamics within the dark space. (a) Scattering of a pump photon into the $\sigma_{-}$-cavity mode; (b) scattering of a pump photon into the $\sigma_{+}$-cavity mode; (c) polarization flip of a cavity photon.

one [Fig. 4(a)]. The process repeats until the atom reaches the Zeeman ground state with $m_{g}=0$. As will be analyzed in more detail below, with two polarizations present the final state of the adiabatic passage still has the atom in $m_{g}=0$. However, two additional processes contribute to the dynamics within the dark eigenspace of energy $E=0$. In one process, a linearly polarized photon from the pump field is scattered into the $\sigma_{+}$-polarized cavity mode, accompanied by a decrease in the magnetic quantum number of the atom [Fig. 4(b)]. As a secondary consequence, the system has to go through an additional cycle of the type shown in Fig. 4(a) to reach the final atomic ground state $m_{g}=0$. Effectively the system has gone through a loop in the atomic level scheme, creating a pair of $\sigma_{+} / \sigma_{-}$photons in the process. Depending on the system parameters, several of these loops may occur. Clearly, loop processes must be avoided if a pure number state is to be generated, as they lead to superposition states of different (and somewhat uncontrollable) photon numbers in the cavity.

The second additional process is the destruction of a $\sigma_{+}$ photon followed by the creation of a $\sigma_{-}$photon (and conversely) with no pump photons involved [Fig. 4(c)]. In this case the photon number in the cavity is not changed, but the polarization of one photon is flipped. At the same time the atom changes its Zeeman substate by two. Due to this process, states beyond the $m_{g}=0$ Zeeman ground state may become temporarily occupied, which is impossible in the single polarization case. The branching ratio determining the relative contribution of each of the three processes of Fig. 4 (at resonance) depends on three factors: the occupation of the cavity mode (influencing induced emission), the ClebschGordan coefficients of the relevant transitions (cf. Fig. 2), and the temporal structure of the couplings and fields. By preparing the atom in the lowest Zeeman state it is guaranteed that the first transition is of type (a), enhancing the probability for further emissions with the desired $\sigma_{-}$polarization. The Clebsch-Gordan coefficients also favor transitions towards decreasing $\left|m_{g}\right|$ so that the adiabatic passage method with two polarizations is still expected to work, albeit with an efficiency degraded by the processes of Figs. 4(b,c). To obtain pure Fock states of the cavity field for a given initial Zeeman substate, a method to suppress these undesired transitions must be found. We will demonstrate in the following that a finite detuning of the cavity from resonance effectively achieves this end.

To analyze the structure of the dark space manifold, it is convenient to parametrize the vectors spanning the $E=0$ eigenspace by the number of photons contained. As photons are transferred to the cavity during adiabatic passage, the initial occupation of the modes (when the pump field $\Omega=0$ and the atom is in the state $g_{-3}$ ) is used to characterize each eigenstate unambiguously. Only such states with an even initial photon number contribute, corresponding to the fact that nonadiabatic coupling to states with higher photon number only occurs by the loop process described above (which adds two photons to the system). For example, for the initial atomic state $\left|g_{-3}\right\rangle$, explicit expressions for the eigenstates corresponding to total initial photon numbers zero, two, and four are given by

$$
\begin{aligned}
\left|E_{0}\right\rangle= & \mathcal{N}_{0}\left[-\sqrt{15} g^{3}(t)\left|g_{-3}, 0,0\right\rangle+\sqrt{45} g^{2}(t) \Omega(t)\left|g_{-2}, 0,1\right\rangle\right. \\
- & \left.\sqrt{18} g(t) \Omega^{2}(t)\left|g_{-1}, 0,2\right\rangle+\Omega^{3}(t)\left|g_{0}, 0,3\right\rangle\right], \\
\left|E_{1}\right\rangle= & \mathcal{N}_{1}\left[\sqrt{60} g^{3}(t)\left|g_{-3}, 1,1\right\rangle-\sqrt{90} g^{2}(t) \Omega(t)\left|g_{-2}, 1,2\right\rangle\right. \\
& +\sqrt{24} g(t) \Omega^{2}(t)\left|g_{-1}, 1,3\right\rangle-\Omega^{3}(t)\left|g_{0}, 1,4\right\rangle \\
& +\sqrt{18} g^{3}(t)\left|g_{-1}, 0,2\right\rangle-\sqrt{36} g^{2}(t) \Omega(t)\left|g_{0}, 0,3\right\rangle \\
& \left.+\sqrt{6} g(t) \Omega^{2}(t)\left|g_{+1}, 0,4\right\rangle\right], \\
\left|E_{2}\right\rangle= & \mathcal{N}_{2}\left[-\sqrt{150} g^{3}(t)\left|g_{-3}, 2,2\right\rangle\right. \\
& +\sqrt{150} g^{2}(t) \Omega(t)\left|g_{-2}, 2,3\right\rangle \\
& -\sqrt{30} g(t) \Omega^{2}(t)\left|g_{-1}, 2,4\right\rangle+\Omega^{3}(t)\left|g_{0}, 2,5\right\rangle \\
& -\sqrt{60} g^{3}(t)\left|g_{-1}, 1,3\right\rangle+\sqrt{90} g^{2}(t) \Omega(t)\left|g_{0}, 1,4\right\rangle \\
& -\sqrt{12} g(t) \Omega^{2}(t)\left|g_{+1}, 1,5\right\rangle-\sqrt{15} g(t)^{3}\left|g_{+1}, 0,4\right\rangle \\
& \left.+\sqrt{15} g(t)^{2} \Omega(t)\left|g_{+2}, 0,5\right\rangle\right],
\end{aligned}
$$

with the symbols $\mathcal{N}_{k}$ denoting normalization constants. Note that $\left|E_{0}\right\rangle$ is identical to the single polarization dark state of Eq. (5).

The evolution of the first two eigenstates $\left(\left|E_{0}\right\rangle\right.$ and $\left.\left|E_{1}\right\rangle\right)$ in terms of the basis state components $\left|g_{m}, n_{+}, n_{-}\right\rangle$is plotted in Fig. 5. As the adiabatic passage proceeds, each state acquires exactly three additional $\sigma_{-}$photons due to the process of Fig. 4(a). Moreover, occupation is redistributed by the polarization flips shown in Fig. 4(c). For a change of eigenstate to occur, the system must undergo a loop process involving the generation of a $\sigma_{+} / \sigma_{-}$photon pair. These latter events are the reason for the failure of standard adiabatic passage to produce a pure Fock state in the twopolarization case with degenerate frequencies.

Possible final states of the system may be deduced from Eqs. (6) by taking the limit $g(t) \rightarrow 0$. As expected from the vanishing coupling of the pump field in the central Zeeman state, the atom emerges from the adiabatic passage always in the $m=0$ ground state as in the single polarization case. This is an important fact, because it ensures that the atom is not 


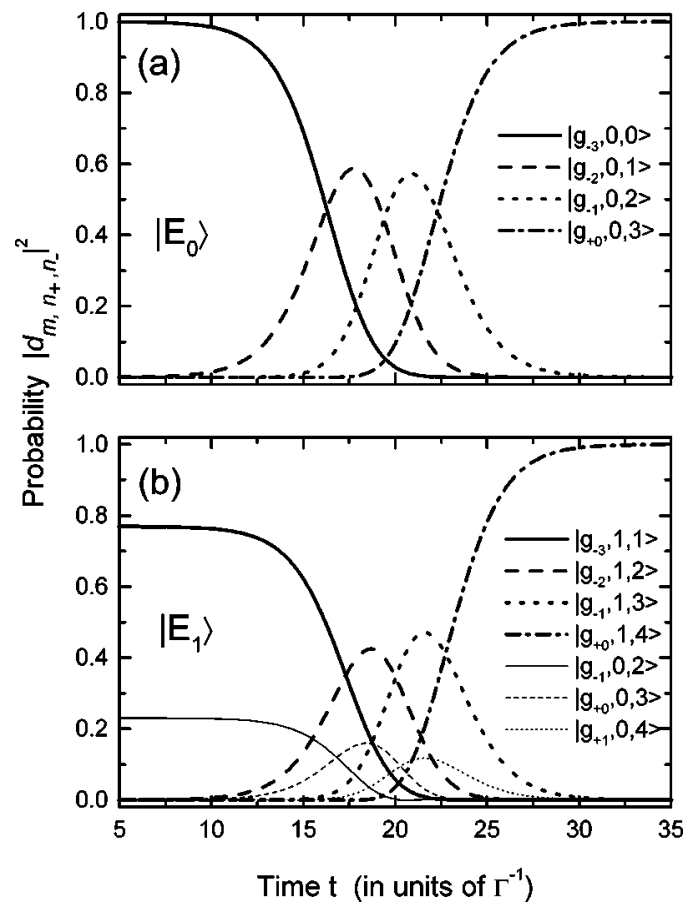

FIG. 5. Time evolution of the first two eigenvectors with degenerate eigenvalues $(E=0)$ in terms of the basis state coefficients $\left|d_{m, n_{+}, n_{-}}\right|^{2}$. They are defined by the expansion $\left|E_{k}(t)\right\rangle$ $=\sum_{m, n \pm} d_{m, n_{+}, n_{-}}^{(k)}(t)\left|g_{m}, n_{+}, n_{-}\right\rangle$[cf. Eq. (6)]. (a) Eigenvector with no $\sigma_{+}$contribution, corresponding to the dark state of the singlepolarization case; (b) eigenvector with one $\sigma_{+}$photon in the cavity; note the additional contributions obtained by polarization flips from $\sigma_{+}$to $\sigma_{-}$(thin lines).

entangled with the field. The cavity modes are found in a superposition of the states $|0,3\rangle,|1,4\rangle,|2,5\rangle, \ldots$, so that the general expression for the final state of the system is

$$
\left|g_{0}\right\rangle \otimes \sum_{k=0}^{\infty} c_{k}|k, 3+k\rangle
$$

Here, the notation $\left|n_{+}, n_{-}\right\rangle$is used to denote number states of the two-mode cavity field.

As a consequence of Eq. (7), along with the desired $\sigma_{-}$ Fock state $|0,3\rangle$, there is a finite probability for additional pairs of $\sigma_{+}$and $\sigma_{-}$photons in the cavity field. The size of those contributions, i.e., the modulus of the coefficients $c_{k}$ in Eq. (7), must be determined from the dynamics generated by $H_{\text {int }}$ (see Sec. IV). However, an estimate for $\left|c_{1}\right|^{2}$ can be obtained by restricting the system to the subspace spanned by the two lowest eigenvectors of the dark space, with $\left|E_{0}\right\rangle$ being the state initially occupied and $\left|E_{1}\right\rangle$ the first state with a $\sigma_{+}$-polarization contribution. As the eigenvectors in Eqs. (6) are time dependent, diabatic Landau-Zener-type transitions between the associated eigenstates occur. The transition probability is obtained by integrating the Schrödinger equation for a two-level system with time-dependent basis states:

$$
\left(i \hbar \frac{\partial}{\partial t}-H_{\text {int }}\right)\left[c_{0}(t)\left|E_{0}(t)\right\rangle+c_{1}(t)\left|E_{1}(t)\right\rangle\right]=0 .
$$

Starting in state $\left|E_{0}\right\rangle$ at $t \rightarrow-\infty$, we derive the probability $\left|c_{1}\right|^{2}$ for a diabatic transition to state $\left|E_{1}\right\rangle$ at $t \rightarrow \infty$ to be given by the following expression:

$$
\begin{aligned}
\left|c_{1}\right|^{2}= & \left(\sin \mid \int_{-\infty}^{+\infty} \frac{\left\langle E_{1}\left|\frac{\partial}{\partial t}\right| E_{0}\right\rangle}{\sqrt{1-\left|\left\langle E_{1} \mid E_{0}\right\rangle\right|^{2}}}\right. \\
& \left.\times e^{-i\left[E_{1}(\tau)-E_{0}(\tau)\right] \tau / \hbar} d \tau \mid\right)^{2} .
\end{aligned}
$$

From Eqs. (6) one finds that the matrix elements appearing under the integral do not vanish, as the eigenvectors and their derivatives are not orthogonal [because of the assumed classical field for $\Omega(t)$ ]. The size of the relevant scalar products as a function of time and hence the diabatic transition probability peak in the middle of the atomic transit. For the parameters of Fig. 3, there is a $22 \%$ probability for the system to undergo a Landau-Zener transition during the adiabatic passage and end up in the state $\left|g_{0}, 1,4\right\rangle$. The model of two degenerate dark states thus demonstrates that adiabatic passage as described in [20] is not well suited to generate number states in a cavity if the two polarizations of the cavity field are closely spaced in frequency.

For the discussion of a method to suppress diabatic transitions to states with the wrong polarization, it is important to note the dependence of the integral in Eq. (9) on the phase factor. When $\left|E_{0}\right\rangle$ and $\left|E_{1}\right\rangle$ are degenerate, this phase factor is obviously zero. There is, however, a simple way to lift this degeneracy: a small detuning $\delta$ of the cavity with respect to the pump field and the atomic transition frequency (assumed to be equal for both polarization modes, i.e., $\delta_{+}=\delta_{-}=\delta$ ) will split the dark space levels into nondegenerate eigenstates, according to their total photon number. Therefore, these new eigenstates are exactly the states given by Eq. (6). The corresponding dark space eigenvalues form a series of energy levels separated by approximately $2 \delta$, since neighboring levels differ by two photons. Through adiabatic passage each state acquires exactly three photons, so that all eigenvalues undergo an increase of $3 \delta$ during the atom's transit through the cavity. This is apparent in the central part of Fig. 6.

When the degeneracy of the dark space is thereby lifted, the phase factor apppearing in Eq. (9) no longer vanishes, but leads to oscillations of the function under the integral. As a consequence, the transition probability $\left|c_{1}\right|^{2}$ will drop when $\delta$ is increased. This simple model is in excellent agreement with the results of a numerical integration of the full interaction Hamiltonian (2).

With the transition rate strongly suppressed at large $\delta$, a system prepared in the $\left|g_{-3}, 0,0\right\rangle$ state will adiabatically follow the $\left|E_{0}\right\rangle$ dressed state in the dark space and end up in the $\left|g_{0}, 0,3\right\rangle$ state, i.e., a Fock state of the cavity field with pure $\sigma_{-}$polarization. In fact, the structure of the phase factor in Eq. (9) implies a new adiabaticity condition for the energy splitting of the dark state manifold and the time $T$ for which the interaction is effective: 


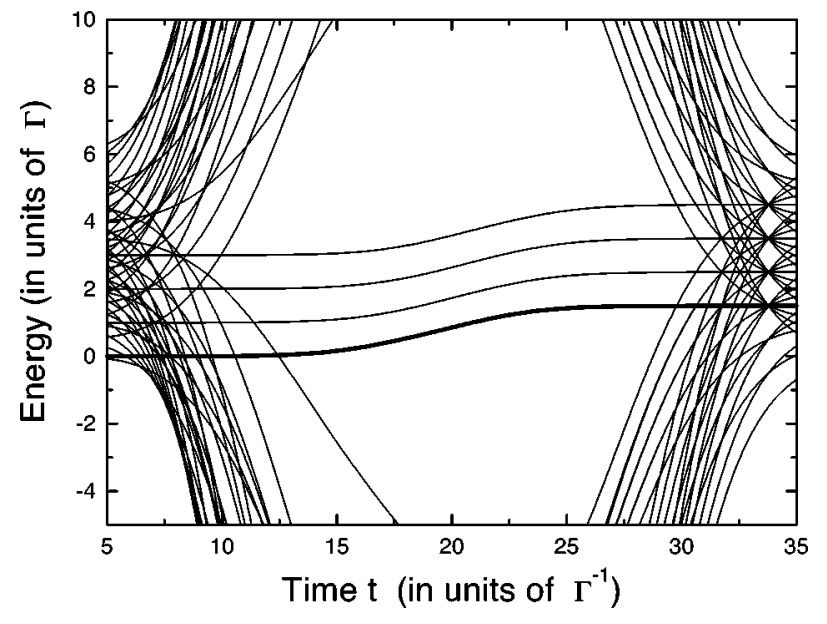

FIG. 6. Energy eigenvalues of $H_{\text {int }}$ as a function of transit time for $\delta=0.5 \Gamma$. The other parameters are the same as in Fig. 3. The bold line represents the single-polarization dark state $\left|E_{0}\right\rangle$. While at the center the splitting of the dark space manifold is well separated from all other levels, the level structure at the edges is rather complex. Note the degeneracy at $\Gamma t \approx 34$.

$$
\left|E_{1}-E_{0}\right| T \approx 2|\delta| T \gg 1
$$

This requirement must be met in addition to the usual (single-polarization) conditions for adiabatic following $[20,34]$

$$
\Omega_{0} T \gg 1, \quad 2 g_{0} \sqrt{n_{ \pm}+1} T \gg 1
$$

which minimize the probability for diabatic transitions to ac-shifted (nondark) dressed states. Since for typical parameters $\delta \ll \Omega_{0}, g_{0}$, the new condition (10) is more stringent than the standard adiabatic condition (11), so that considerably slower atoms (by a factor of $g_{0} / \delta$ ) are needed in the two-polarization case.

In the discussion, so far it was assumed implicitly that the energies of nondark dressed states (ac-Stark shifted on the order of $g_{0}$ and $\Omega_{0}$ ) and the dark space eigenvalues (on a scale of $|\delta|$ ) are well separated, as is the case in the middle of the atomic transit. However, this assumption is not valid at the leading edge of the cavity mode and the trailing edge of the pump field, where the ac-Stark shifts of the energy levels become comparable to the splitting of the dark states $(g(t), \Omega(t) \approx|\delta|)$. In this regime, a complex structure of the eigenvalues ensues, as is apparent from the edges of the dressed-state diagram of Fig. 6. An enlarged view of the energy levels for the atom entering the cavity mode (Fig. 7) shows that the lower dark state $\left|E_{0}\right\rangle$, which is initially occupied, undergoes a large number of crossings with nondark levels. Most of these crossings are actually avoided, as illustrated in the blow up of the first crossing in Fig. 7. Since the synthesis of pure Fock states requires that the system follows the lowest dark state, transitions to any intersecting states must be avoided, i.e., all crossings must be passed diabatically. This condition puts an upper bound on the products appearing in Eqs. (10), (11), limiting them to a finite range of values.

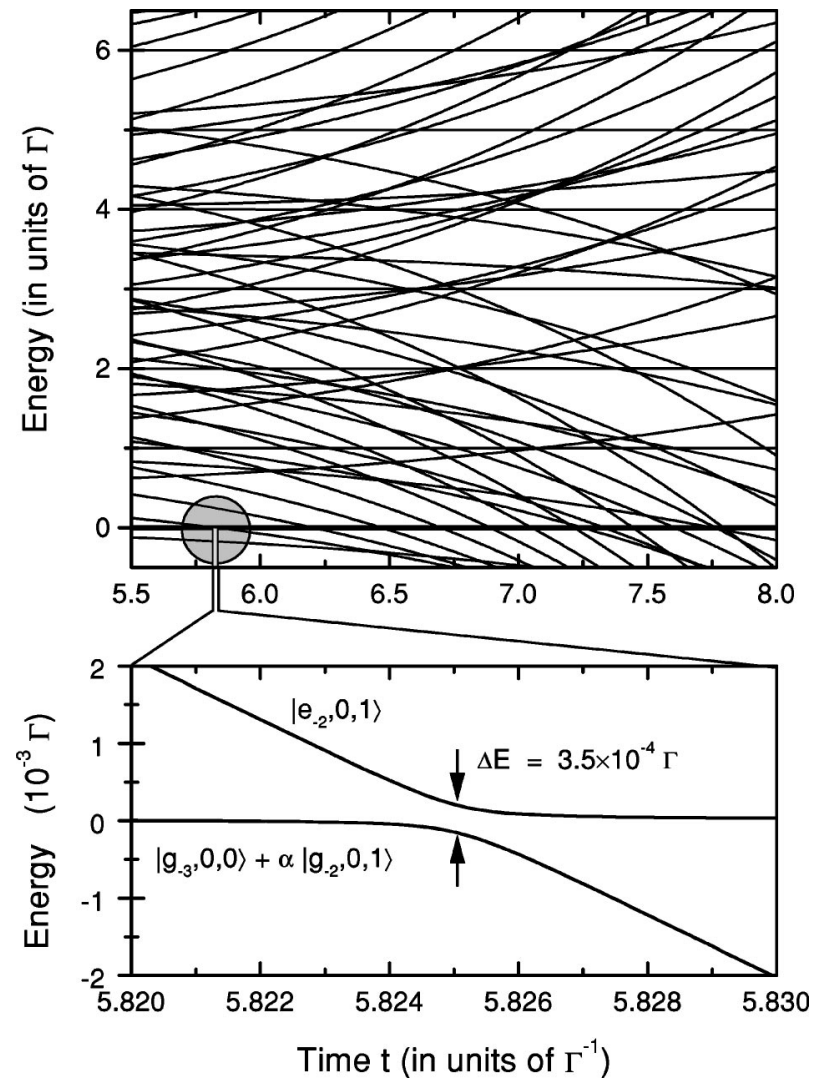

FIG. 7. Close-up view of energy eigenvalues of $H_{\text {int }}$ at the leading edge of the cavity mode for the parameters of Fig. 6. The enlargement of the first crossing of the lowest dark state (with state $\left.\left|e_{-2}, 0,1\right\rangle\right)$ shows that the crossing is actually avoided on a very fine scale.

A quantitative evaluation of the eigenvalue spectra shows that the effects of level crossings are different when the atom is mainly exposed to the cavity field (in the initial phase of the atomic transit with $g / \Omega \gg 1$ ) and when the interaction is primarily with the pump field (in the final phase with $g / \Omega$ $\ll 1)$. In the former case, it should be noted that only twolevel crossings occur. The largest level splitting is found at the first crossing, which is the one shown in the lower part of Fig. 7. Corresponding to the small coupling at the edge of the cavity mode, it has an energy splitting orders of magnitude smaller than $\Gamma\left(3.5 \times 10^{-4} \Gamma\right.$ in the example of Fig. 7$)$. In order to pass this crossing adiabatically, the atom would have to have a velocity $10^{6}$ times lower than that taken for Fig. 3. Therefore, for realistic atomic velocities the diabatic condition is easily fulfilled on entry and the atom will pass the crossings as if the levels were not coupled. This result is confirmed by the numerical calculations of the following section.

A different situation occurs at the trailing edge of the pump field. At a time $t$ such that the classical Rabi frequency has decreased to $\Omega(t)=4|\delta|$, the ac-shifted dressed levels become degenerate with the series of dark states (neglecting coupling to the cavity mode), as is apparent from Fig. 6 and shown in more detail in Fig. 8 for the lowest dark state. The phenomenon is found in the single- [Fig. 8(a)] and the twopolarization case [Fig. 8(b)] at finite detuning. The degen- 


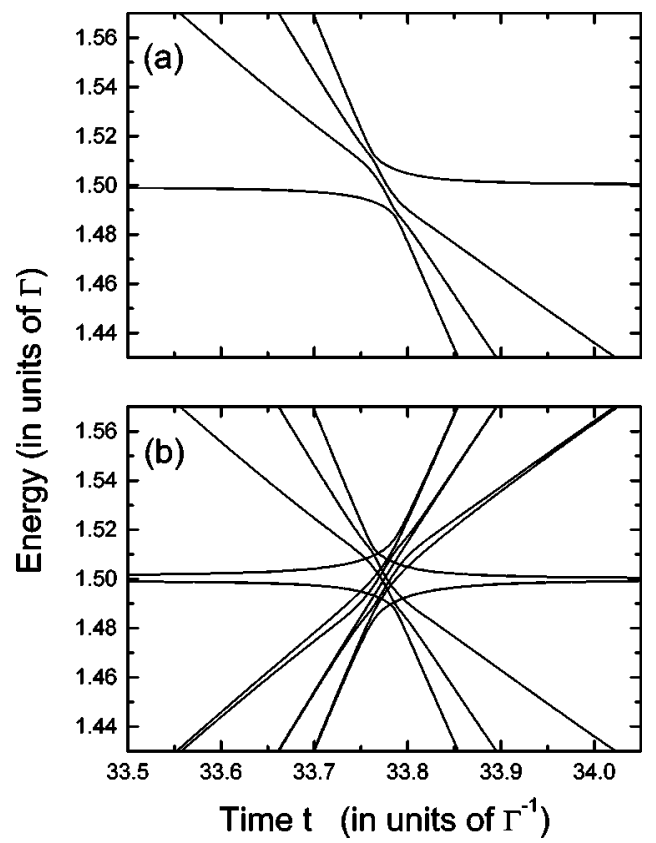

FIG. 8. Dressed level structure at the trailing edge of the pump pulse where $\Omega(t) \approx 4|\delta|$. A degeneracy of the atom-pump interaction leads to a multilevel avoided crossing. (a) Single-polarization case; (b) two-polarization case. The parameters are the same as in Fig. 6.

eracy is lifted due to the interaction with the cavity mode(s), so that a multiple-level avoided crossing is created. For positive detuning $\delta$ and a single polarization, the multiplicity is given by the number of dressed states with positive ac-Stark shift [three levels crossing the dark state in the example of Fig. 8(a)]. In order to emerge from the crossing in the dark state, the system would have to undergo diabatic jumps across three levels with a substantial energy splitting (on the order of $0.02 \Gamma$ for $\delta=0.5 \Gamma$ in the example of Fig. 8). Consequently, there is a finite probability for transitions to states outside the dark state manifold. These partly adiabatic transitions at the trailing edge of the pump field limit the detuning $\delta$ that may be applied to achieve adiabatic conditions at the center of the interaction region. They also set an upper limit on the transit time $T$ and hence on the products of Eqs. (10) and (11), as pointed out earlier.

For two polarizations, an additional degeneracy occurs due to contributions from states with higher photon number. In this case dressed states with negative and positive Stark shift are involved in the crossing [Fig. 8(b)]. Note that a similar multiple-level crossing does not occur when the atom enters the cavity, as the dressed energies of the quantized cavity mode are nondegenerate due to their dependence on the photon number. Hence the restrictions for detuning and time of flight are more stringent during interaction with the classical field in the final phase of the atomic transit.

In the following sections, the preceding conclusions drawn from the eigenvalue spectrum of the Hamiltonian in Eq. (2) and a simple two-level model will be put on a quantitative basis by integrating the master equation of the system, including dissipative processes in the treatment. A suitable method is the quantum Monte Carlo wave-function technique. The results will be employed to describe a strategy and calculate the efficiency for the synthesis of pure $|0,3\rangle$ or $|3,0\rangle$ states of the cavity field or superpositions of both.

\section{QUANTUM MONTE CARLO SIMULATIONS}

In the previous analysis only the coherent interaction of the atom and the two cavity modes was considered. A realistic model of the system also has to take into account dissipation that enters due to spontaneous decay of the atom and damping of the cavity mode. The dissipative atom-cavity system is described by the following master equation for the density operator $\rho(t)$ of the system:

$$
\begin{aligned}
\frac{\partial \rho}{\partial t}= & -\frac{i}{\hbar}\left[H_{\mathrm{eff}}, \rho\right]+2 \kappa\left(a_{+} \rho a_{+}^{\dagger}+a_{-} \rho a_{-}^{\dagger}\right) \\
& +\Gamma \sum_{\sigma=0, \pm 1} A_{\sigma} \rho A_{\sigma}^{\dagger} .
\end{aligned}
$$

Here, $H_{\text {eff }}$ is a non-Hermitian effective Hamiltonian including decay terms given by

$$
H_{\mathrm{eff}}=H_{\mathrm{int}}-i \kappa\left(a_{+}^{\dagger} a_{+}+a_{-}^{\dagger} a_{-}\right)-i \frac{\Gamma}{2} \sum_{\sigma=0, \pm 1} A_{\sigma}^{\dagger} A_{\sigma} .
$$

Solutions of the master equation may be obtained by integrating Eq. (12) numerically. However, the size of the Hilbert space accessible to the system in the two-polarization case is rather large, even if its basis is truncated at moderate photon numbers. For example, for atomic levels with $F_{g}$ $=F_{e}=3$ and a cutoff photon number of seven in both cavity modes, the dimension $N$ of the Hilbert space is $2 \times(2 \times 3$ $+1) \times 8 \times 8=896$. The computational expense involved in density matrix calculations, which scales as $N^{4}$, makes integrating the master equation impractical. A more suitable approach is to simulate the master equation by using Monte Carlo wave functions [35-37]. Here, only the Schrödinger equation with the non-Hermitian Hamiltonian $H_{\text {eff }}$ has to be integrated and the problem size scales as $N^{2}$. Dissipation is taken into account by quantum jumps, which occur at random times with a probability determined by the decay rate of the wave-function norm. The jumps are implemented by applying so-called collapse operators to the system wave function and normalizing the result. Between subsequent jumps, the system evolves according to the Schrödinger equation with the non-Hermitian effective Hamiltonian $H_{\text {eff }}$. In this way, one obtains a wave function conditioned on a particular decay history. The system density matrix and operator expectation values are obtained by repeating the calculation many times and averaging over the resulting trajectories. Details of the procedure are found in Refs. [35-38].

In addition to the potential for more efficient use of computational resources, the method offers the benefit that for each of the simulated trajectories a record of the quantum jumps that have occurred during the system evolution is ob- 


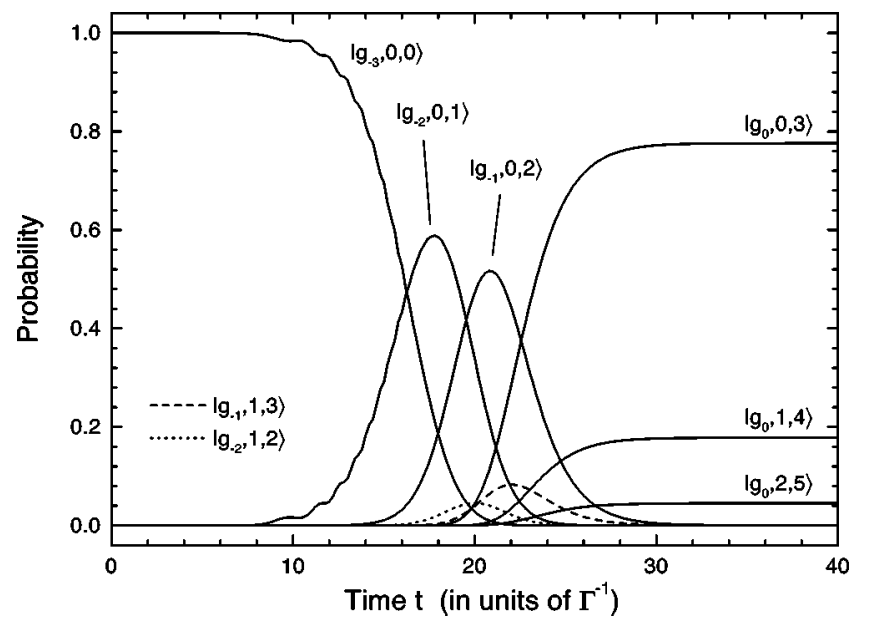

FIG. 9. System evolution of an atom and two degenerate polarization modes, obtained from a Monte Carlo wave-function simulation including atomic spontaneous emission. Shown is the occupation probability of the basis states of the system. The amplitudes of the Gaussian pulses are $g_{0}=25 \Gamma$ (at $t=17 \Gamma^{-1}$ ) and $\Omega_{0}=50 \Gamma$ (at $\left.t=23 \Gamma^{-1}\right)$, with a FWHM of $10 \Gamma^{-1}$. The cavity is at resonance with the pump field $\left(\delta_{ \pm}=\omega_{c \pm}-\omega_{l}=0\right)$.

tained. In the case of Eq. (12), there are five possible collapse operators and hence five types of jumps given by

$$
\sqrt{2 \kappa} a_{ \pm}, \quad \sqrt{\Gamma} A_{\sigma}, \quad \sigma=0, \pm 1
$$

corresponding to decay of the two orthogonally polarized cavity modes and spontaneous emission of a photon with polarization $\sigma$, respectively. Decay of the cavity modes may be associated with photodetection events in an individual experimental run. Note that, when the polarization of the photons leaking from the cavity is analyzed in a basis different from that of the intracavity field (as set by the spatial quantization of the atom), linear combinations of the field decay operators $a_{ \pm}$must be employed [39], which will be used in Sec. VI to analyze GHZ correlations between cavity photons.

In a first set of simulations, only atomic decay is included, while cavity decay is still neglected. Ideally, as long as the evolution of the system is restricted to the dark space, the influence of spontaneous emission on the dynamics should be weak, since the upper atomic levels are not excited. Hence, the conclusions of Sec. III should apply to the numerical results in this regime.

We start by studying the system evolution for an $F_{g}=3$ $\rightarrow F_{e}=3$ transition in the case of atom, cavity, and laser fields on resonance $(\delta=0)$, i.e., the case of a degenerate dark space. The initial state is again $\left|g_{-3}, 0,0\right\rangle$. Figure 9 shows the system evolution during the passage of an atom through the time-dependent cavity coupling $g(t)$ and the laser field $\Omega(t)$ in terms of the basis state occupation. The probability maximum is shifted in turn from the initial state to $\left|g_{-2}, 0,1\right\rangle$ to $\left|g_{-1}, 0,2\right\rangle$, before finally reaching $\left|g_{0}, 0,3\right\rangle$. These are exactly the levels comprised by the lowest dressed state $\left|E_{0}\right\rangle$ in the dark space manifold of Eq. (6), which should be the only ones occupied if the interaction was en-

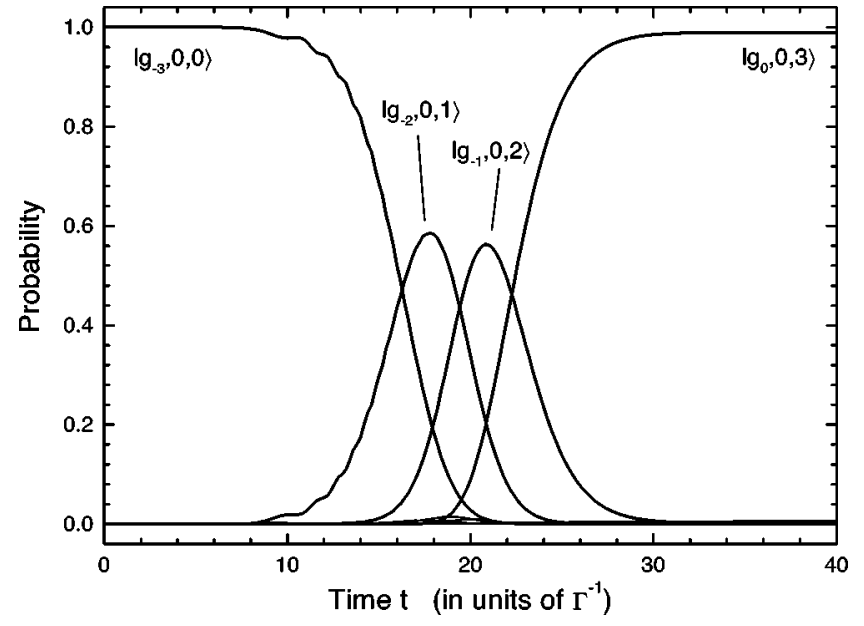

FIG. 10. System evolution of atom and two polarization modes, at a finite cavity detuning $\delta=0.6 \Gamma$, showing nearly ideal adiabatic passage. The amplitudes and timing of the applied pulses are the same as in Fig. 9.

tirely adiabatic. Approximately halfway through the transit, however, contributions from levels $\left|g_{0}, 1,4\right\rangle$ and $\left|g_{0}, 2,5\right\rangle$ start to appear. These are due to the diabatic transitions to states with higher photon number predicted from the twolevel model analyzed in Sec. III. At the end of the transit, the atom is found in state $\left|g_{0}\right\rangle$ and the cavity is in a superposition of the states $|0,3\rangle,|1,4\rangle$, and $|2,5\rangle$, etc., instead of the desired pure Fock state $|0,3\rangle$, confirming the earlier result of Eq. (7).

In the two-level model of Eqs. (8), (9) in Sec. III, a finite cavity detuning $\delta$ was shown to lift the degeneracy of the dark space and lead to a strong suppression of diabatic transitions. What remains to be investigated is the question of whether this mechanism is still effective, if the full system dynamics including atomic decay and level crossings in the entrance and exit phase of the interaction are taken into account. In Fig. 10, a Monte Carlo calculation of the system evolution for a cavity detuning $\delta_{ \pm}=0.6 \Gamma$ is shown. The pump field is still assumed to be on resonance with the atomic frequency $\left(\omega_{l}=\omega_{a}\right)$. As can be seen from the resulting final state, the finite cavity detuning leads to the desired strong suppression of states with $\sigma_{+}$photons and an amplitude for the $\sigma_{-}$Fock state, which is close to unity (99\%). The atom remains in the lowest branch of the dark state manifold and is adiabatically transferred from $\left|g_{-3}, 0,0\right\rangle$ to $\left|g_{0}, 0,3\right\rangle$. Therefore, passage through a detuned cavity is adiabatic and well suited to generate a Fock state of one polarization of the cavity field, even for a cavity supporting two degenerate polarization modes. By choosing other initial states $\left(\left|g_{-m}, 0,0\right\rangle, m=1,2\right)$, states with photon number $m$ $<F_{g}$ may be obtained as well. Coherent superpositions of Zeeman ground states with $0<m \leqslant F_{g}$ are also possible, leading to superpositions of photon number states. Thus an operationally simple modification of the work of Ref. [20] suffices to make a workable scheme for the generation of " arbitrary" field states for a single polarization mode.

To study systematically the effect of cavity detuning $\delta$ (assumed to be equal for both polarizations, i.e., $\delta_{+}=\delta_{-}$ 


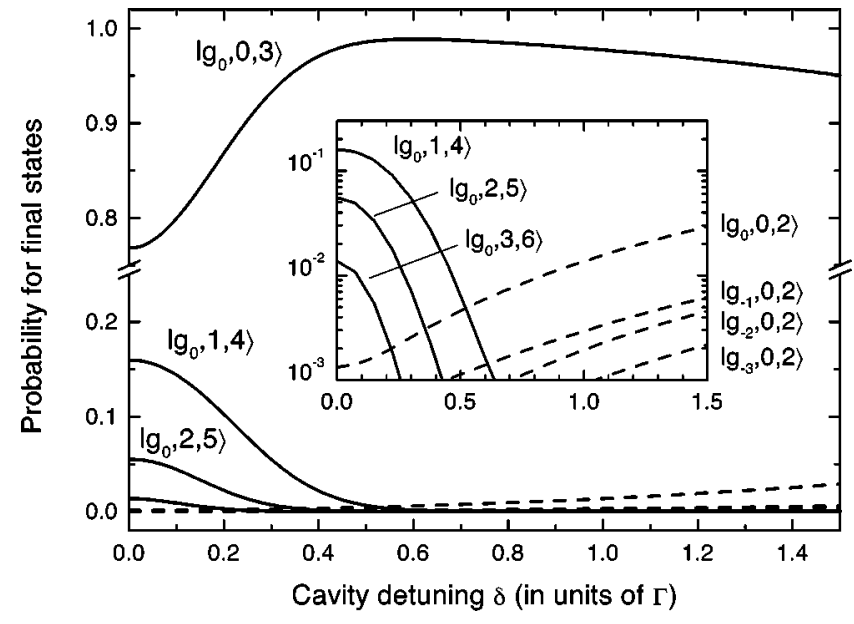

FIG. 11. Final state probabilities as a function of cavity detuning $\delta_{+}=\delta_{-}=\delta$, showing the transition to an adiabatic regime for $\delta$ $>0.5 \Gamma$. The parameters correspond to those of Fig. 9 .

$=\delta$ ) on the efficiency of our scheme, we have calculated the final state as a function of $\delta$, with the result displayed in Fig. 11. As $\delta$ is increased from zero, the probability for contributions from basis states with the "wrong" cavity polarization $\left(\sigma_{+}\right)$drops to zero, while the pure $\sigma_{-}$state becomes occupied with $99 \%$ efficiency around $\delta \approx 0.6 \Gamma$.

In contrast to the results of the two-level model, the probability for a pure $\sigma_{-}$Fock state drops again slightly when $\delta$ is increased to values larger than $0.6 \Gamma$. The reason is the dynamics in the initial and the final phase of the atomic transit (for example, at the multilevel crossing shown in Fig. 8 ), which becomes partly adiabatic if $\delta$ is chosen too large. As a consequence, population is transferred to levels outside the dark state manifold, leading not only to a finite probability for a decreased photon number in the cavity, but also to entanglement between the atom and the cavity field. This is illustrated in Fig. 11 by the rise of contributions from levels $\left|g_{k}, 0,2\right\rangle(k=-3, \ldots, 0)$ as $\delta$ is increased beyond $0.6 \Gamma$. Note that this limitation of adiabatic passage also occurs in the single-polarization case and is not connected to the structure of the dark space.

A blown-up plot of the probability for reaching the final state $\left|g_{0}, 0,3\right\rangle$ vs detuning $\delta$ is presented in Fig. 12. The results for three different values of the coupling constant $g_{0}$ show that the efficiency of the method approaches unity when the coupling is increased $\left(99.6 \%\right.$ at $\left.g_{0}=50 \Gamma\right)$. The optimum detuning is $\delta \approx 0.6 \Gamma$ and depends on the coupling only weakly. For comparison, the single-polarization probability for a three-photon Fock state is also included in Fig. 12. The large deviation between the two sets of curves for values below $\delta=0.6 \Gamma$ clearly shows the inadequacy of the single-polarization model. For large detunings, on the other hand, the results coincide, demonstrating that in this regime the two-polarization dynamics is well described by the adiabatic following of a single dark state. The common asymptotic behavior of the curves is another hint to the polarization independent mechanism populating nondark states at the multilevel avoided crossing occurring for large $\delta$ (Fig. 8). Figure 12 suggests that, for an optimum value of $\delta$, the

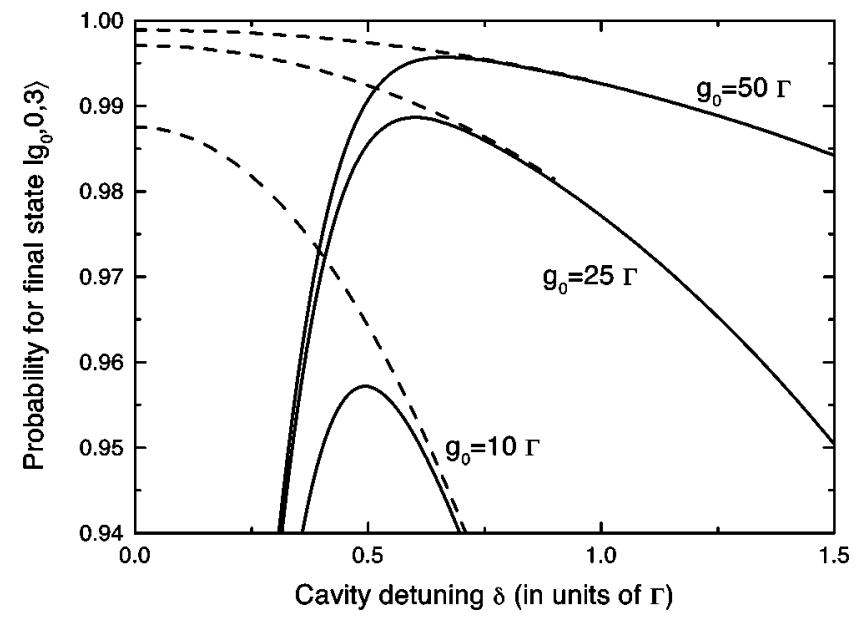

FIG. 12. Probability for a final state $\left|g_{0}, 0,3\right\rangle$ as a function of cavity detuning $\delta$ for three coupling constants in the case of twopolarization adiabatic passage (solid lines). The single-polarization $\left(\sigma_{-}\right)$results are included as well (dotted lines). The timing of the pulses is the same as in Fig. 9 and $\Omega_{0}=2 g_{0}$. Note that, for this figure, the full master equation was simulated in order to obtain an accuracy well below $1 \%$.

purity of the final state is only limited by the atom-cavity coupling $g_{0}$ that may be achieved experimentally, if the interaction time is held constant.

In the calculations presented so far, the detunings of the two polarization modes of the cavity were always assumed to be equal $\left(\delta_{+}=\delta_{-}\right)$. In order to probe the sensitivity of the system to a finite mode splitting, which may occur due to cavity imperfections, we have varied the detuning $\delta_{+}$of the $\sigma_{+}$-polarized mode while keeping the $\sigma_{-}$-polarized mode at resonance with the atom $\left(\delta_{-}=0\right)$. Again, for the initial state $\left|g_{-3}, 0,0\right\rangle$, Fig. 13 shows the probability to reach a final state $\left|g_{0}, 0,3\right\rangle$ as a function of $\delta_{+}$. It exhibits a gradual transition from the diabatic losses in the degenerate two-polarization case to the perfect adiabatic passage obtained for a single

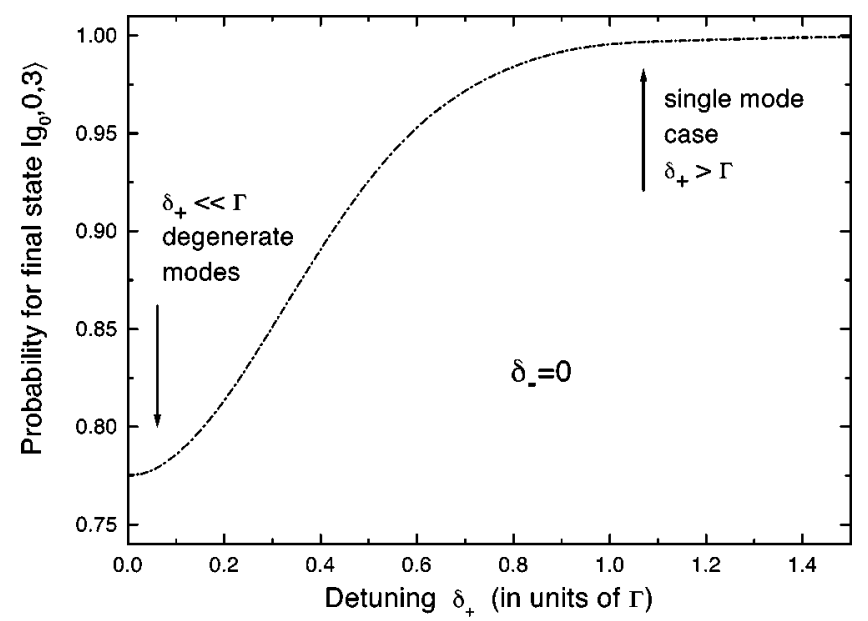

FIG. 13. Probability for a final state $\left|g_{0}, 0,3\right\rangle$ as a function of the detuning $\delta_{+}$of the $\sigma_{+}$-polarized cavity mode. The $\sigma_{-}$mode is assumed to be on resonance and a coupling $g_{0}=25 \Gamma$ was used for both modes. The timing of the pulses is the same as in Fig. 9. 
$\left(\sigma_{-}\right)$polarization, as $\delta_{+}$is increased from zero. The single mode description applies when the $\sigma_{+}$mode is far enough detuned from resonance for its influence on the system dynamics to be neglected, which is the case for detunings $\delta_{+}$ $\geq \Gamma$. Figure 13 suggests that, for the model of degenerate modes to be valid, the splitting should be small compared with the natural linewidth $\Gamma$. As long as $\delta_{+}<0.1 \Gamma$, the final state probability deviates from the degenerate case by less than a percent. Note that for realistic cavities a well-defined splitting in the $\sigma_{ \pm}$basis may be difficult to achieve, because of a lack of control over the polarization basis in which birefringence of the mirrors occurs.

At fixed coupling $g_{0}$, the efficiency of the state synthesis scheme may be increased by extending the time $T$ during which the atom interacts with the cavity mode and the pump field, i.e., using slower atoms for the adiabatic passage. In Sec. III it was found that adiabatic transitions to nondark states place an upper limit on $T$. There is, however, another restriction for the interaction time, which is set by the decay time of the cavity modes. This will be demonstrated in the following section by including cavity decay in the master equation.

\section{PREPARATION OF A GREENBERGER-HORNE- ZEILINGER STATE}

Adiabatic passage with a detuned cavity and an atom prepared in the $\left|g_{-3}\right\rangle$ level was shown in Sec. IV to synthesize Fock states of $\sigma_{-}$polarization and effectively suppress excitation of the $\sigma_{+}$-polarized cavity mode. An important property of the proposed scheme is that it is entirely symmetric under a change of sign in the magnetic quantum number and a simultaneous exchange of $\sigma_{-}$and $\sigma_{+}$polarization, as is apparent from the master Eq. (12) and the relevant Clebsch-Gordan coefficients. If, for example, the system is prepared in the state $\left|g_{+3}, 0,0\right\rangle$, a Fock state with three $\sigma_{+}$ photons in the cavity $(|3,0\rangle)$ will be generated. Another notable feature of the two polarization adiabatic passage for an $F \rightarrow F$ transition is that at the end of the interaction the atom is always in the $m_{g}=0$ Zeeman ground state $\left(\left|g_{0}\right\rangle\right)$, independent of the initially occupied Zeeman substates. Therefore, the atomic part of the wave function is always disentangled from the state of the cavity field and can be disregarded without introducing projection noise to the measurement of the cavity field. The method is not restricted to single Zeeman levels as initial atomic states. The atom may be prepared in a coherent superposition of Zeeman ground states, leading to superposition states of the cavity field as described for the single-polarization model in Refs. [20,23]. A particularly intriguing possibility in the two-polarization case is to use coherent superpositions of Zeeman sublevels with different sign of the magnetic quantum number $m$. In this case, quantum superpositions of photon states with different polarization are synthesized, i.e., the adiabatic passage maps the Zeeman coherence of the atomic state to polarization entanglement of the two cavity modes.

An important example of such field states are the number states of $n$ photons maximally entangled in polarization, which are obtained for a superposition state of $n$ photons in the $\sigma_{+}$mode and $n$ photons in the $\sigma_{-}$mode. With the notation previously introduced for a two-mode cavity field, these states may be written as

$$
\left|\Psi_{n}\right\rangle \equiv \frac{1}{\sqrt{2}}(|n, 0\rangle+|0, n\rangle)
$$

For the state $\left|\Psi_{2}\right\rangle$, there is a close correspondence with the correlated two-particle states used to test Bell's inequalities $[1,40]$ obtained from local hidden-variable theories. Numerous experiments have shown these inequalities to be violated [41]. Yet even more interesting properties emerge from the entangled state $\left|\Psi_{3}\right\rangle$ of three particles, corresponding to a so-called GHZ state, as was introduced by Greenberger, Horne, and Zeilinger [3]. Its significance is based on the fact that, to test theories of local realism against quantum mechanics, a single set of observations of this state is sufficient ("all or nothing"). This is in contrast to the statistical nature of the violation of Bell's inequalities (for $n=2$ ).

There is a notable difference between the states $(|n, 0\rangle$ $+|0, n\rangle)$ considered here and the original Bell-type states of the form $\left(\left|+{ }_{1},+_{2}, \ldots,+{ }_{n}\right\rangle+\left|-{ }_{1},-{ }_{2}, \ldots,-{ }_{n}\right\rangle\right)$. While in the former case all photons of a given polarization occupy a single mode, in the latter case they are distributed over $n$ distinct (i.e., distinguishable) modes $(1,2, \ldots, n)$. However, as will be discussed below, we restrict our attention to the subensemble of photoelectric detection events that produce one and only one detection event ("click") at each of $n$ sets of detectors. Hence, from the perspective of a post diction, the state $\left|\Psi_{n}\right\rangle$ for the subensemble of distinct "temporal" modes generates correlations identical to those of the Belltype states. More generally, by tailoring the interaction profiles $g(t)$ and $\Omega(t)$ of cavity and pump field, an improved timing of cavity decay events may be achieved, so that in fact successive quanta $1,2, \ldots, n$ would emerge from the cavity in well defined intervals and would be routed in a predefined fashion to respective detectors, making the requirement of separate detection events even less restrictive.

In the following, we give a detailed description of how the two-polarization adiabatic passage scheme may be extended to generate a GHZ-type state $\left|\Psi_{3}\right\rangle$ (denoted as $|\mathrm{GHZ}\rangle$ ) with high efficiency. Towards this end the $F_{g}=3$ $\rightarrow F_{e}=3$ transition used for the generation of the singlepolarization Fock state will be employed again. Note that states $\left|\Psi_{n}\right\rangle$ with $n>3$ may be synthesized as well, if an atomic transition with a high enough angular momentum were to be used. For the $F_{g}=4 \rightarrow F_{e}=4$ hyperfine transition in cesium, for example, the state $\left|\Psi_{4}\right\rangle$ may be generated in exactly the same way as $\left|\Psi_{3}\right\rangle$.

The starting point for the generation of a GHZ-type state is to create a coherent superposition of the two outer Zeeman ground states $\left(m_{g}= \pm 3\right)$. This task may be achieved by using suitably timed microwave [27] or Raman pulses [28]. Ideal adiabatic passage of an atom through the cavity modes with coupling $g(t)$ and the pump field $\Omega(t)$, either by physical motion or by suitable modulation of the coupling for a 


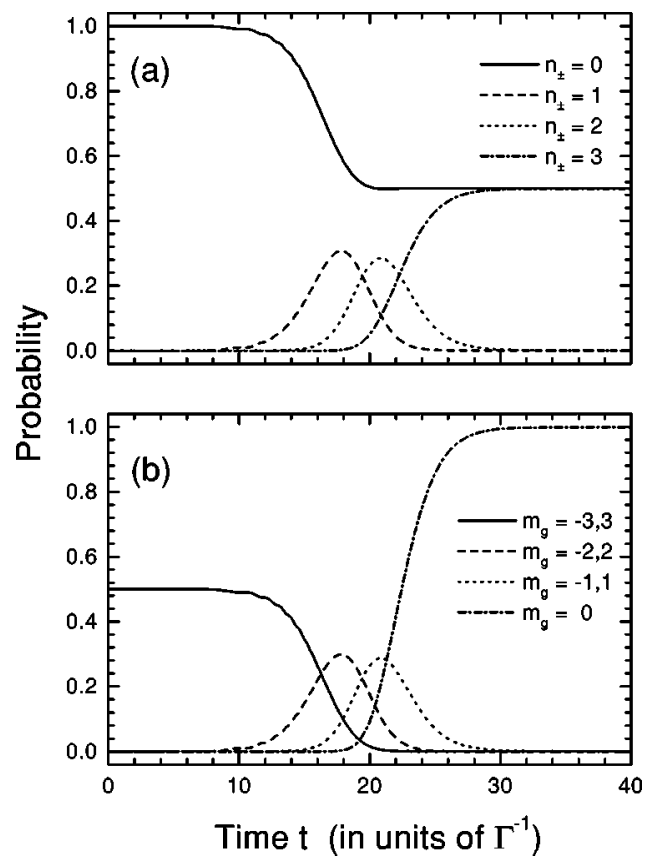

FIG. 14. Monte Carlo simulation of the preparation of a GHZtype state in the absence of cavity decay. The amplitudes of the cavity and pump field are $g_{0}=25 \Gamma$ (at $t=17 \Gamma^{-1}$ ) and $\Omega_{0}=50 \Gamma$ (at $t=23 \Gamma^{-1}$ ) with a FWHM of $10 \Gamma^{-1}$, the cavity detuning is $\delta$ $=0.6 \Gamma$, and the initial preparation of the atom is a coherent superposition of $m_{g}=3$ and $m_{g}=-3$. (a) Occupation of the cavity modes (the distributions are identical for the $\sigma_{+}$and the $\sigma_{-}$mode); (b) atomic ground-state populations (the curves for $+m_{g}$ and $-m_{g}$ coincide). The final state is a $99 \%$ pure state $|\mathrm{GHZ}\rangle$.

stationary atom in the cavity, would then generate the following mapping of ground-state Zeeman coherences to the cavity field:

$$
\begin{aligned}
\left(\left|g_{-3}\right\rangle+\left|g_{+3}\right\rangle\right) \otimes|0,0\rangle & \Rightarrow\left|g_{0}\right\rangle \otimes(|0,3\rangle+|3,0\rangle) \\
& \sim\left|g_{0}\right\rangle \otimes|\mathrm{GHZ}\rangle
\end{aligned}
$$

and would thus leave the cavity in a maximally entangled state of the GHZ-type. The transitions relevant for this synthesis scheme are the ones shown in Fig. 2(b).

We have tested the efficiency of such a GHZ-state preparation procedure numerically by simulating the relevant master equation using Monte Carlo wave functions, again neglecting cavity decay in the first instance but with atomic decay included. The same parameters as in Secs. III and IV are used with a detuning $\delta=0.6 \Gamma$ to achieve optimum purity of the final state. The resulting averaged time evolution is shown in Fig. 14. In Fig. 14(a) the occupation of the cavity mode states is plotted, while Fig. 14(b) shows the corresponding atomic ground-state populations. As expected due to the symmetry of the system, the time-dependent photon distribution of the two cavity modes is identical. The same is true for the occupation of Zeeman ground-state levels with the same modulus $\left|m_{g}\right|$. At the end of the adiabatic passage, there is almost perfect polarization entanglement with a $99 \%$ probability for the cavity to be found in the GHZ state $(|0,3\rangle+|3,0\rangle)$ and the atom in state $\left|g_{0}\right\rangle$. In correspondence

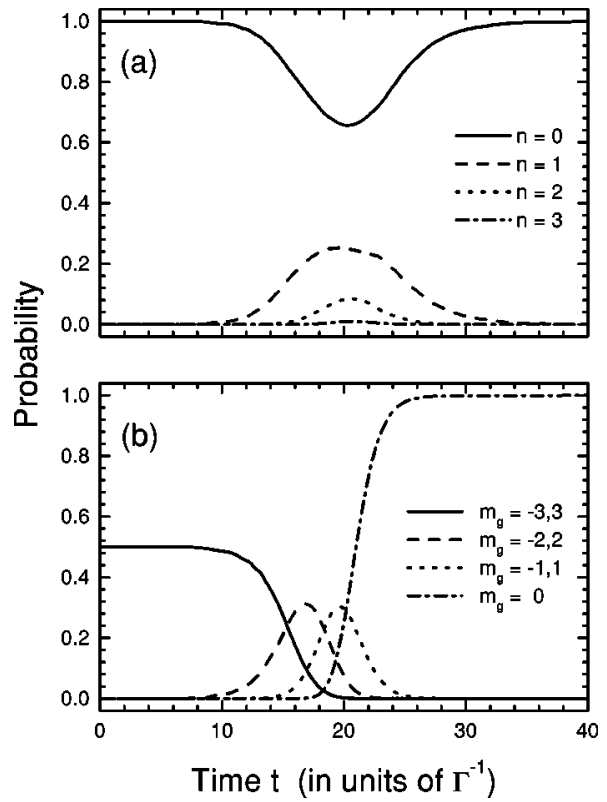

FIG. 15. Monte Carlo simulation of adiabatic passage for the same parameters as in Fig. 14, but with a cavity decay rate $\kappa$ $=0.2 \Gamma$. (a) Occupation of the cavity modes; (b) atomic groundstate populations.

with the discussion of Sec. III, contributions of higher terms in the dark space manifold, which are again of the form $(|k, 3+k\rangle+|3+k, k\rangle), k=1,2, \ldots$, are strongly suppressed. As mentioned above, the efficiency could be further increased by using an even stronger coupling $g_{0}$ or slower atoms.

Beyond these encouraging results, a realistic simulation of our experiment has to take into account dissipation of the cavity field, which has been neglected so far. In recent experiments cavity decay rates $\kappa / 2 \pi$ ranging from 0.6 to 35 $\mathrm{MHz}$ have been achieved [29,31-33]. As a point of reference, here we take initially $\kappa / 2 \pi=1 \mathrm{MHz}$, resulting in $\kappa / \Gamma=0.2$, although we will subsequently investigate the dependence of the state generation on $\kappa$. The cavity decay time $1 / \kappa$ is then comparable to the adiabatic passage time scale of maximum efficiency for state generation [cf. Fig. 3(a) for the time dependence of $g(t)$ and $\Omega(t)$ ], so that cavity decay will play an important role in the present scheme and must be considered in the simulation.

We have repeated the Monto Carlo wave-function simulation of the GHZ-state generation of Fig. 14 using the full master equation (12), including the cavity decay terms with $\kappa=0.2 \Gamma$. In Fig. 15, the resulting average time evolution of the cavity mode occupations and the atomic ground-state populations is displayed. As expected, cavity decay strongly affects the dynamics of the field modes. During the adiabatic passage, the probability for the excitation of photon states with $n>0$ is substantially reduced compared to the case without cavity decay. The probability for one photon in a cavity mode never exceeds $25 \%$. When the atom leaves the cavity, both modes of the cavity field have already decayed to the $n=0$ ground state at the rate $\kappa^{-1}$ [Fig. 15(a)]. Obviously, there is no time when the cavity modes approach a pure GHZ-type state. This result is in correspondence with 
previous studies of the generation of quantum superpositions in a lossy single-polarization cavity [20,23].

Perhaps surprisingly, Fig. 15(b) shows a completely different behavior for the atomic population. The occupation of the Zeeman states is almost unaffected by the presence of cavity decay. The evolution proceeds essentially undisturbed in close correspondence to the perfect cavity case shown in Fig. 14(b). The fact that cavity dissipation does not affect the atomic dynamics during the adiabatic passage was also observed in the single-polarization calculations of Ref. [23]. It implies that atomic coherence is transferred without "damage" as the cavity field undergoes a quantum jump to a lower dark state manifold [42]. The preservation of atomic coherence during the adiabatic passage even for a lossy cavity is an essential requirement for the detection of GHZ correlations between the photons leaking from the cavity, as will be explained in the following section.

\section{DETECTION OF GHZ CORRELATIONS}

In principle, there are two different approaches to detecting nonclassical states of the cavity field. First, the field inside the resonator may be probed directly. This may be achieved, for example, by sending a second atom through the cavity and reversing the adiabatic passage scheme to map the photon number distribution of the cavity field to coherences in the Zeeman substructure $[20,23,43]$. Measurements of the atomic substates would then provide information on the state of both field modes (in principle the "complete" state [43]). The required timing of the pump and probe atoms would certainly be difficult to achieve for an atomic beam (with Poissonian fluctuations). However, in the optical domain, the most severe obstacle to this approach is cavity decay, since, as shown above, dissipation of the cavity field prevents the modes from ever reaching a pure GHZ state, so that any attempt to observe a signature of that state would fail.

This state of affairs suggests observing the photons leaking from the cavity mirrors during decay of the field as the method of choice for detecting GHZ correlations, especially since it affords the opportunity for tests of local realism with the "flying" photons that emerge from the cavity. Correlations between these emitted photons could readily be measured by coincidence detection, with these measurements performed in spatially separated regions as required by the locality assumption.

Before presenting a more detailed proposal for an experimental setup to probe the decay photons, we will first discuss the expected correlations between photons emerging from a lossy cavity. For simplicity we start by assuming that the two cavity modes already are in the state $|\mathrm{GHZ}\rangle$ given by Eq. (15) for $n=3$. Afterwards we will examine how the correlations are affected if dissipation is present during the state synthesis.

Greenberger, Horne, and Zeilinger have developed their argument for three arbitrary particles in a maximally entangled state. Therefore, their analysis can also be applied to three photons in the state $|0,3\rangle+|3,0\rangle$. To obtain an operator with two eigenvalues \pm 1 , as considered by GHZ, we define an observable equivalent to the spin of an atomic system. It is easy to verify that the following operators satisfy the spin commutation relations

$$
L_{+} \equiv a_{+}^{\dagger} a_{-}, \quad L_{-} \equiv a_{-}^{\dagger} a_{+}, \quad L_{z} \equiv \frac{1}{2}\left(a_{+}^{\dagger} a_{+}-a_{-}^{\dagger} a_{-}\right) .
$$

The operators $L_{ \pm}$perform a change of polarization on a cavity photon, which is analogous to a spin flip. The observable corresponding to the particle analyzer in the setup proposed by GHZ is then given by the superposition of $L_{+}$and $L_{-}$ with a variable relative phase shift $\phi$, namely,

$$
L(\phi) \equiv e^{i \phi / 2} L_{+}+e^{-i \phi / 2} L_{-},
$$

with the required eigenvalues \pm 1 . To arrive at a measurement procedure for $L(\phi)$ in the case of photons emitted from the cavity, we first rewrite

$$
L(\phi)=a_{x}^{\dagger}(\phi) a_{x}(\phi)-a_{y}^{\dagger}(\phi) a_{y}(\phi) .
$$

The operators $a_{x}(\phi)$ and $a_{y}(\phi)$ are annihilation operators of linearly polarized photons in the $x$ and $y$ directions of a coordinate system rotated by an angle $\phi / 4$. They are related to the cavity mode operators by

$$
\begin{aligned}
& a_{x}(\phi)=\frac{1}{\sqrt{2}}\left(e^{-i \phi / 4} a_{+}+e^{i \phi / 4} a_{-}\right), \\
& a_{y}(\phi)=\frac{i}{\sqrt{2}}\left(e^{-i \phi / 4} a_{+}-e^{i \phi / 4} a_{-}\right) .
\end{aligned}
$$

Therefore, according to Eq. (19), $L(\phi)$ can be determined by measuring the difference in the occupation number of two orthogonal linear polarization modes in a rotated frame.

To be consistent with the GHZ gedanken experiment, we must make the additional assumption that each of the three photons from the state $|\mathrm{GHZ}\rangle$ is delivered to a separate analyzer. In this case there are only two possible outcomes of a measurement of $L(\phi)$ : a value of +1 is obtained if an $x$-polarized photon is detected, a value of -1 for a $y$-polarized photon.

Figure 16 shows an idealized experimental setup for the detection of GHZ correlations. We assume well-defined generation times for each of the three photons, as might be achieved in principle by a succession of suitable shifts applied to the atomic levels in concert with a somewhat revised time history for $g(t)$ and $\Omega(t)$ during the adiabatic passage. Each photon emitted from the cavity is then directed to one of three analyzers. In front of each analyzer $(k=1,2,3)$ there is a half-wave plate rotating the respective linear polarization basis by angle $\phi_{k} / 4$. The polarization $\left[x\left(\phi_{k}\right)\right.$ or $\left.y\left(\phi_{k}\right)\right]$ is measured with a linear polarizing beamsplitter (PBS) and photon counters at the two output ports. The analyzers are assumed to be positioned far from the cavity and from each other, so that the individual measurements may be regarded as causally separated.

To arrive at the correlations discussed by Greenberger, Horne, and Zeilinger, the product of the three detector results 


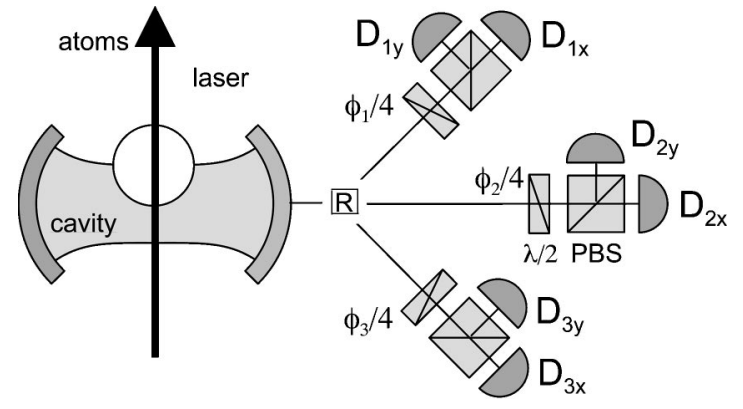

FIG. 16. Idealized experimental setup for the detection of GHZ correlations between photons leaking from the output port of the cavity. Each of the three decay photons is sent by router $\mathrm{R}$ to a particular analyzer, consisting of a half-wave plate, a polarizing beamsplitter PBS, and a pair of detectors $D_{i \xi}$ counting photons of linear polarization $\xi(\xi=x, y)$ in the basis determined by the wave plate.

is considered. It is straightforward to calculate its expectation value if the photons are in the GHZ state

$$
\left\langle\mathrm{GHZ}\left|L_{1}\left(\phi_{1}\right) L_{2}\left(\phi_{2}\right) L_{3}\left(\phi_{3}\right)\right| \mathrm{GHZ}\right\rangle=\cos \left(\phi_{1}+\phi_{2}+\phi_{3}\right),
$$

where again the subscript identifies the analyzer.

The most interesting situation occurs when the detector angles are set such that $\left(\phi_{1}+\phi_{2}+\phi_{3}\right)$ is an integer multiple of $\pi$. In this case the average value is either +1 or -1 , which coincides with the maximum and minimum possible value, respectively, since any individual product of detector outcomes is either +1 or -1 . This implies that each individual measurement of triple correlations yields the same result. In this case the photon triplets show perfect correlations in a sense that knowing the results of two detectors allows one to predict the outcome of the third measurement with certainty.

In Ref. [3] it was shown that the triple correlations of particles prepared in a GHZ state violate theories based on the assumption of local realism in a rather striking way. This may be demonstrated by considering the correlations for the following choice of detector angles:

$$
\begin{gathered}
\left\langle\mathrm{GHZ}\left|L_{1}\left(\frac{\pi}{2}\right) L_{2}\left(\frac{\pi}{2}\right) L_{3}(0)\right| \mathrm{GHZ}\right\rangle=-1, \\
\left\langle\mathrm{GHZ}\left|L_{1}\left(\frac{\pi}{2}\right) L_{2}(0) L_{3}\left(\frac{\pi}{2}\right)\right| \mathrm{GHZ}\right\rangle=-1, \\
\left\langle\mathrm{GHZ}\left|L_{1}(0) L_{2}\left(\frac{\pi}{2}\right) L_{3}\left(\frac{\pi}{2}\right)\right| \mathrm{GHZ}\right\rangle=-1, \\
\left\langle\mathrm{GHZ}\left|L_{1}(0) L_{2}(0) L_{3}(0)\right| \mathrm{GHZ}\right\rangle=+1 .
\end{gathered}
$$

If the result of each detector was independent of the angles $\phi_{k}$ of the other detectors (as required by the locality assumption for widely separated detectors), the expectation values of Eqs. (22) would factor into the product of three functions $A_{k}\left(\phi_{k}\right)$ that can take values \pm 1 :

$$
\begin{gathered}
\left\langle\mathrm{GHZ}\left|L_{1}\left(\phi_{1}\right) L_{2}\left(\phi_{2}\right) L_{3}\left(\phi_{3}\right)\right| \mathrm{GHZ}\right\rangle \\
=A_{1}\left(\phi_{1}\right) A_{2}\left(\phi_{2}\right) A_{3}\left(\phi_{3}\right) .
\end{gathered}
$$

Multiplying the four left-hand sides of the set of Eqs. (22), a product expression is obtained in which every factor $A_{k}(\phi)$ $(k=1 \ldots 3 ; \phi=0, \pi / 2)$ appears exactly twice. Therefore, using $A_{k}^{2}=1$, a result of 1 is expected for the overall left-hand side product, if local realism holds. On the other hand, the right-hand sides of Eqs. (22), which were obtained quantum mechanically, multiply to give -1 , clearly contradicting local realism.

We now return to the situation encountered in our experiment for realistic parameters of coupling, transit time, and cavity decay time. It has been shown in Sec. V that, even for the best Fabry-Pérot cavities available [44] (excluding for the moment the whispering gallery modes of quartz microspheres [45-47]), decay may occur well before the adiabatic passage is terminated so that the intracavity field never reaches the state $|\mathrm{GHZ}\rangle$. Further, since there is not a welldefined time history for the times of the photon emissions, the operator expectation value (21) is not necessarily a relevant quantity. However, correlations between the photons emitted during adiabatic passage may still be probed. The important question then becomes whether or not they show a similar behavior as that described by Eq. (21).

The Monte Carlo wave-function method offers a convenient way to evaluate correlations between photons emitted from the cavity. For each simulated trajectory a classical record of the quantum jumps, which have occured during the adiabatic passage, is kept that contains information on the polarization of the decay photon corresponding to the jump. Averaging over many runs, photon-counting distributions and correlation functions for the detectors involved are readily calculated. To distinguish between the six possible detection channels (two for each of the three analyzers), the collapse operators for the two cavity modes, which appear in Eq. (12), must be replaced by a new set of annihilation and creation operators. As each decay of the cavity field can be associated with a click in a certain detector, six new collapse operators are needed. They involve the operators defined in Eq. (20) in the rotated linear polarization bases:

$$
\begin{gathered}
\sqrt{2 \kappa} a_{x}\left(\phi_{k}\right)=\sqrt{\kappa}\left(a_{+} e^{-i \phi_{k} / 4}+a_{-} e^{i \phi_{k} / 4}\right), \\
\sqrt{2 \kappa} a_{y}\left(\phi_{k}\right)=i \sqrt{\kappa}\left(a_{+} e^{-i \phi_{k} / 4-a_{-} e^{i \phi_{k} / 4}}\right), \quad k=1,2,3 .
\end{gathered}
$$

The new master equation to simulate is

$$
\begin{aligned}
\frac{\partial \rho}{\partial t}= & -\frac{i}{\hbar}\left[H_{\mathrm{eff}}, \rho\right]+\frac{2 \kappa}{3} \sum_{k=1,2,3}\left[a_{x}\left(\phi_{k}\right) \rho a_{x}^{\dagger}\left(\phi_{k}\right)\right. \\
& \left.+a_{y}\left(\phi_{k}\right) \rho a_{y}^{\dagger}\left(\phi_{k}\right)\right]+\Gamma \sum_{\sigma=0, \pm 1} A_{\sigma} \rho A_{\sigma}^{\dagger} .
\end{aligned}
$$

In the original GHZ proposal, three apertures are used to select the subset of particles emitted in the direction of the three analyzers. In our case all fields are emitted into the same spatial mode, so that one has to rely on beamsplitters to 


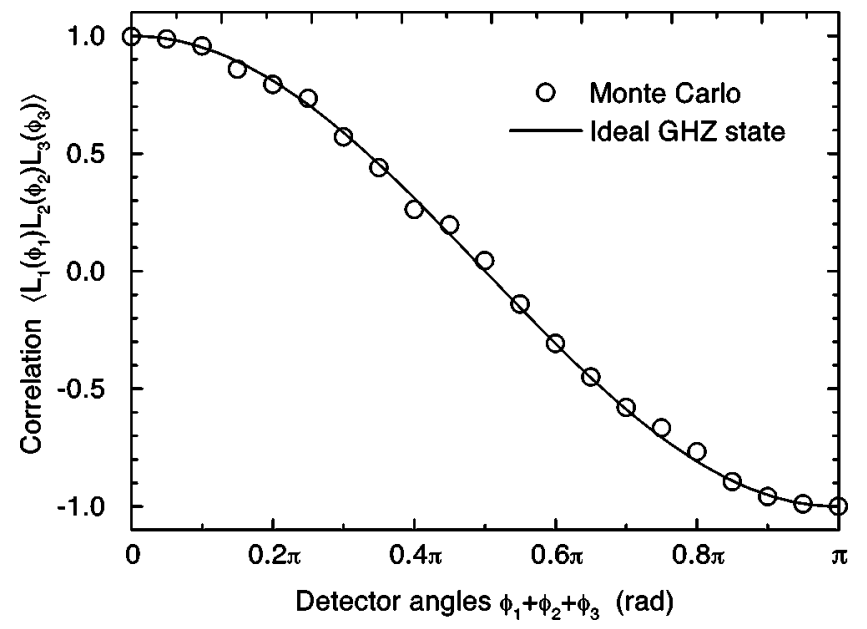

FIG. 17. Average triple correlations of photons decaying from the cavity, obtained from the quantum jump record of a Monte Carlo wave-function simulation. The adiabatic passage parameters are chosen as in Fig. 14, but with a finite cavity decay rate $\kappa$ $=0.1 \Gamma$. All three analyzers are set at the same angle $\left(\phi_{1}=\phi_{2}\right.$ $=\phi_{3}$ ), but equivalent results are obtained for different orientations. The solid line represents the correlations expected for an ideal GHZ state of the cavity field in the absence of decay.

produce three outgoing beams. In such a scheme one cannot guarantee that photons are actually registered by separate analyzers, as required by the GHZ argument. This was taken into account in the simulations by discarding trajectories with a single detector being "hit' more than once. This way we could also discriminate against trajectories, in which more than three photons were detected (due to occasional diabatic transitions within the dark space manifold).

A comparison between the triple correlation function $\left\langle L_{1}(\phi) L_{2}(\phi) L_{3}(\phi)\right\rangle$, obtained by evaluating the quantum jump record of Monte Carlo simulations (with a decay rate $\kappa=0.1 \Gamma$ ), and the result calculated for an ideal GHZ state [Eq. (21)], which would have been generated in the absence of cavity decay, is shown in Fig. 17. Equal angles $\phi$ were chosen for all three detectors. Within the accuracy of the Monte Carlo simulation results, the quantum jump correlations precisely follow the cosine function predicted analytically for a stationary GHZ state. In particular, perfect correlations are found for $\Sigma_{k} \phi_{k}=0$ and $\Sigma_{k} \phi_{k}=\pi$. The Monte Carlo wave-function simulations were repeated for different detector orientations. Whenever the sum over the angles was an integer multiple of $\pi$, every trajectory with exactly three photons detected showed the correlations expected from a GHZ state, even when the field started leaking from the cavity well before the last photon was deposited to the cavity modes by the atom.

It is remarkable that GHZ-type correlations are present between the decay photons even when the average occupation of the cavity mode is far below one. The observed correlations must be attributed to the dynamics of the adiabatic passage and cannot be the property of a certain quantum state of the cavity field [such as the case in Eq. (15)]. Preservation of coherence between subsequent photon decays is clearly required and is provided by the Zeeman ground states, which

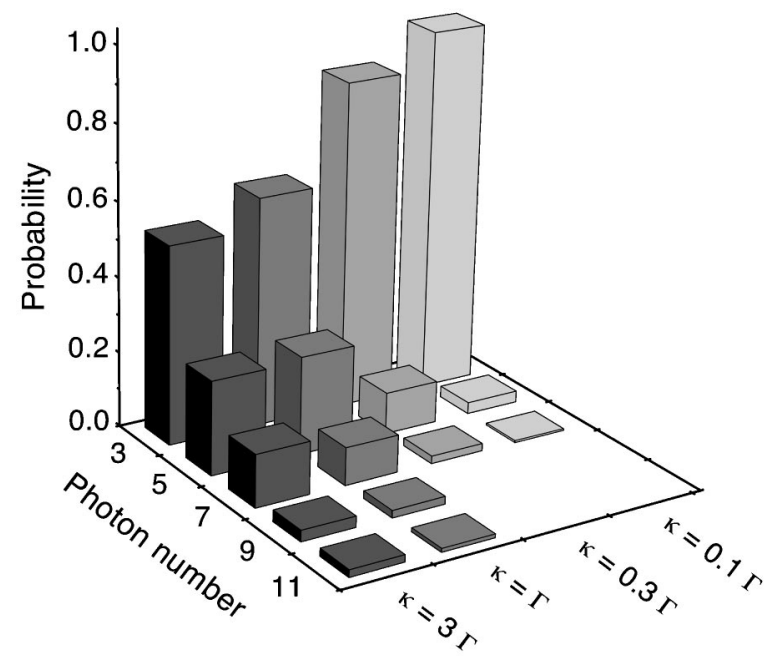

FIG. 18. Distribution of the number of photons escaping during a single atomic transit for different cavity decay rates. Otherwise, the parameters are the same as in Fig. 14. For faster cavity decay, the probability for higher photon counts grows due to diabatic transitions within the dark state manifold.

are not affected by decay of the cavity field (see the time evolution of the atomic level population in Fig. 15 in Sec. V). The fact that entanglement of the "flying" photons is generated by the system dynamics distinguishes our method from most other proposals for the generation of GHZcorrelated particles. Another notable feature of our scheme is that cavity dissipation does not lead to a loss of coherence, but rather is utilized for the birth of these flying "fields" with GHZ correlations into the external environment.

An important question is the dependence of the triple correlations on the cavity decay time. Simulating the adiabtic passage for different $\kappa$, we found that the appearance of perfect correlations persisted even if $\kappa$ was increased to a value of $10 \Gamma$, as long as only trajectories with exactly three decay photons were considered. However, the number of trajectories with five, seven, or more photons generated by diabatic transitions during the atom's passage was found to grow with $\kappa$. This is illustrated in Fig. 18, showing the distribution of the number of escaped photons for four different cavity decay rates. Obviously, with more than three photons generated in the cavity, no triple correlations can be expected. While it is no problem to discard these trajectories in the numerical simulations, in an actual experiment these processes may lead to wrong results due to finite detection efficiency. If, for example, two out of five emitted photons are not detected, the remaining photons are mistakenly registered as a three-photon event but would show no definite correlations. The probability for these events must therefore be minimized, which can be achieved by using a suitable detuning and strength of the atom-cavity coupling to suppress diabatic transitions during the passage of the atom (as described in Secs. III and IV) and by chosing a small cavity decay rate. Since excited atomic states are occupied with an acceptably small probability only, the system is not compromised by atomic spontaneous emission, which otherwise would also result in the generation of excess photons. 


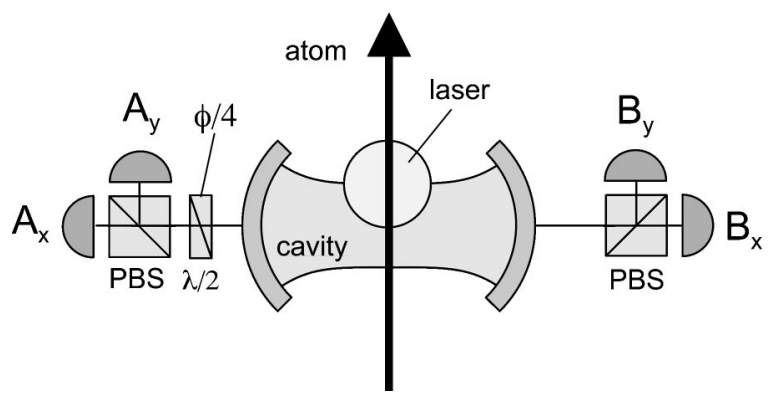

FIG. 19. Simplified setup for a realistic experiment to probe GHZ correlations of photons emitted from a cavity with two output ports. The pair of detectors $A$ analyzes the photon polarization in a rotated linear basis (given by the orientation of the wave plate), while detectors $B$ use a fixed linear basis. The GHZ argument may be tested by observing the result of analyzer $B$ if both detectors $A_{x}$ and $A_{y}$ have registered a photon.

In an actual experiment, one may take advantage of some simplifictions. For the experimental verification of the GHZ argument [cf. Eq. (22)], two of the three analyzers should be set at the same variable angle $\left(\phi_{1}=\phi_{2}=\phi\right)$, while the other is always set at an angle $\phi_{3}=0$. Therefore, it is sufficient to use a pair of analyzers, with a half-wave plate only in front of one of them. Instead of using beamsplitters to route the decay photons, a cavity with two semitransparent output mirrors may be used with an analyzer placed on either side. The proposed setup is shown in Fig. 19. To avoid problems due to detector dead time, events should only be considered if all the photons from the passage of a single atom are registered by different photon counters. If the probability for the generation of more than three photons is negligible (which is the case, for example, for a detuning $\delta \approx 0.6 \Gamma$ and a small cavity decay rate $\kappa \approx 0.1 \Gamma$, cf. Figs. 12 and 18 ), this last condition may be fulfilled by discarding runs with less than three distinct counters clicking.

In this scheme, GHZ correlations could be detected by examining the result of the $B$ counters when both $A$ counters detect a photon. According to Eq. (21), the expectation value for the outcome of the $B$ measurement is then $-\cos (2 \phi)$, as the product of the $A$ results is always negative. Perfect correlations result for $\phi=\pi / 2$, when only the $B_{x}$ counter should receive the third photon and for $\phi=0$, when the third photon should always be registered by $B_{y}$. To maximize the probability for two photons being emitted through the mirror on the $A$ side, and one through the $B$ mirror, the $A$ mirror transmission $T$ should be twice that of the $B$ mirror. In this case, the measurement record would show three separate photoelectric events in the required combination of analyzers in $44.4 \%$ of the atomic transits.

\section{ATOM-PHOTON GHZ CORRELATIONS}

The method proposed in Sec. V for the synthesis of polarization-entangled states of the cavity field requires the initial generation of coherent superpositions of Zeeman ground-state sublevels. While certainly feasible, this requirement adds the need for additional microwave or laser pulse manipulation of the atom before the adiabatic passage. The

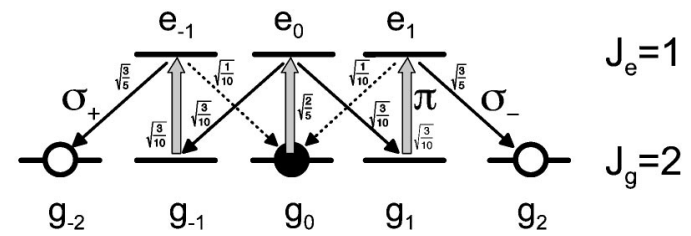

FIG. 20. Level scheme of the $\left(J_{g}=2\right) \rightarrow\left(J_{e}=1\right)$ transition, showing the transitions relevant for an atom prepared in the $m_{g}$ $=0$ Zeeman sublevel (solid circle). The wide arrows indicate the $\pi$-polarized pump transitions. The predominant cavity-induced transitions are shown as solid arrows, the undesired transitions are dotted. After the adiabatic passage, the atom is found in a superposition of the $m_{g}=-2$ and the $m_{g}=+2$ level (open circles), while the cavity contains two photons in a superposition of $\sigma_{+}$and $\sigma_{-}$ polarization.

state synthesis procedure would be greatly simplified if it were possible to generate entangled states starting from a single Zeeman sublevel, which is easily prepared by optical pumping. In this final section we will show that, by using a different Zeeman scheme, it is indeed possible to generate a GHZ-type state with an atom initially pumped to the $m_{g}$ $=0$ ground state. However, in this case one of the entangled particles is the atom itself, which must be detected in order to observe GHZ correlations.

The atomic detection should be efficient, have a good time resolution, and a low background. These requirements are met by using metastable atoms, which can be readily detected with secondary electron multipliers [48,49]. A suitable transition is $1 s_{5}(J=2) \rightarrow 2 p_{10}(J=1)$ in argon, starting from the metastable state $1 s_{5}$, with a transition wavelength of $912.30 \mathrm{~nm}$ and a decay rate $\Gamma / 2 \pi=3.0 \mathrm{MHz}$. Figure 20 shows the corresponding $J=2 \rightarrow J=1$ level scheme. The adiabatic passage procedure again uses a $\pi$-polarized pump field. The atom in this case must be prepared in the $m=0$ level, e.g., by pumping on the $1 s_{5}(J$ $=2) \rightarrow 2 p_{8}(J=2)$ transition, and the cavity modes are assumed to be empty. The lowest state of the dark space manifold that connects to this initial state is

$$
\begin{aligned}
\left|E_{0}\right\rangle= & -\sqrt{12} g^{2}(t)\left|g_{0}, 0,0\right\rangle+2 g(t) \Omega(t)\left(\left|g_{-1}, 1,0\right\rangle\right. \\
& \left.+\left|g_{1}, 0,1\right\rangle\right)-\Omega^{2}(t)\left(\left|g_{-2}, 2,0\right\rangle+\left|g_{2}, 0,2\right\rangle\right) .
\end{aligned}
$$

Due to the symmetry of the interaction Hamiltonian of Eq. (2) with respect to $\sigma_{+} / \sigma_{-}$and positive/negative magnetic quantum numbers $m$, the adiabatic passage proceeds from the initial state to superposition states of positive Zeeman levels with $\sigma_{-}$photons and negative Zeeman levels with $\sigma_{+}$photons. In contrast to the case considered in Sec. V, the adiabatic passage leaves the atom in a state entangled with the cavity field:

$$
\left|g_{0}\right\rangle \otimes|0,0\rangle \Rightarrow\left|g_{-2}, 2,0\right\rangle+\left|g_{2}, 0,2\right\rangle .
$$

As in Sec. IV, diabatic transitions may be suppressed and the system dynamics restricted to adiabatic changes of state (26) by using a finite cavity detuning $\delta$. This is demonstrated by the results of a Monte Carlo wave-function simulation of the system using the parameters for the above specified tran- 


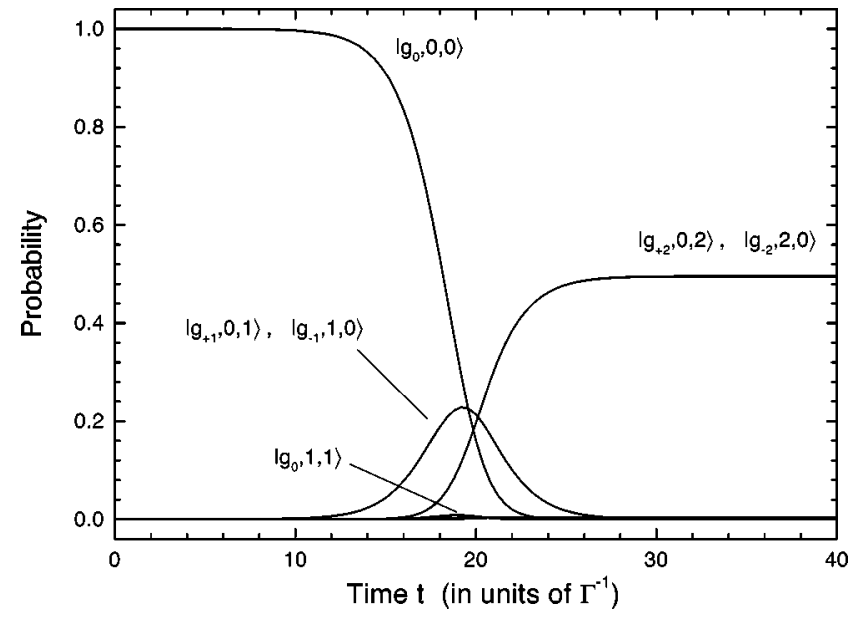

FIG. 21. Monte Carlo wave-function simulation of twopolarization adiabatic passage in the case of a $J=2 \rightarrow J=1$ transition. Atomic spontaneous emission is included, but cavity decay is neglected. The system evolution is given in terms of the occupation probability of the relevant basis states. Note that due to symmetry, the curves for the levels $\left|g_{m}, 0, m\right\rangle$ and $\left|g_{-m}, m, 0\right\rangle$ fall exactly on top of each other. The amplitudes of the Gaussian pulses for metastable argon were chosen as $g_{0}=30 \Gamma$ (at $t=17 \Gamma^{-1}$ ) and $\Omega_{0}$ $=50 \Gamma$ (at $\left.t=23 \Gamma^{-1}\right)$, with FWHM $10 \Gamma^{-1}$. The cavity detuning is $\delta=0.6 \Gamma$.

sition in argon. In Fig. 21, the occupation that is initially in level $\left|g_{0}, 0,0\right\rangle$ is transferred with equal probability to the levels $\left|g_{1}, 1,0\right\rangle,\left|g_{-1}, 0,1\right\rangle$ to end finally in a superposition of the states $\left|g_{2}, 2,0\right\rangle,\left|g_{-2}, 0,2\right\rangle$, where for these calculations cavity decay has been neglected.

The final state in Eq. (27) should also display the correlations discussed by Greenberger, Horne, and Zeilinger, which are independent of the type of particle considered. The cavity decay photons are detected by using analyzers in front of the two cavity output ports as depicted in Fig. 19. The detection of the state-information carried by the atom requires a different strategy. By analogy with the optical case, it should involve the interference of positive and negative Zeeman states and allow for a variable phase shift between both components. A possible experimental realization of such a detector is a Stern-Gerlach analyzer with the magnetic field oriented perpendicular to the cavity axis ( $z$ axis), which is the spatial quantization axis. The required phase shift is then obtained by rotating the magnetic field axis in the $x-y$ plane by an angle $\theta / 4$. A sketch of the proposed setup is shown in Fig. 22. The energy eigenstates in the analyzer are obtained from the following operator involving angular momenta $J_{ \pm}$acting on the ground state

$$
J(\theta)=\frac{1}{2}\left(e^{-i \theta / 4} J_{+}+e^{i \theta / 4} J_{-}\right),
$$

with eigenvalues $m_{J}(\theta)=0, \pm 1, \pm 2$. To establish GHZ correlations between the atom and the photons emitted from the cavity, one considers the operator with an eigenvalue of 1 if the outcome of the Stern-Gerlach measurement is even $\left[m_{J}(\theta)=0, \pm 2\right]$ and an eigenvalue of -1 if it is odd

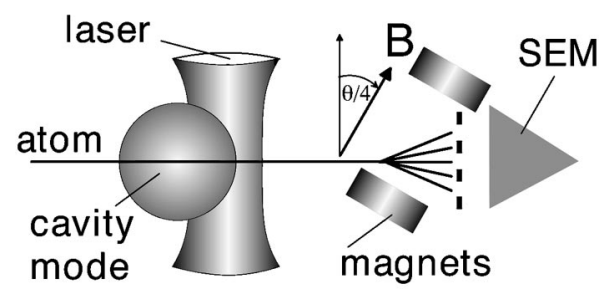

FIG. 22. Experimental setup for the detection of GHZ correlations between two cavity decay photons and the atomic state. The photons are detected as illustrated in Fig. 19. After the adiabatic passage, the atoms traverse a Stern-Gerlach detector with a magnetic field rotated by an angle $\theta / 4$. A grating then selects either the odd or the even orders of the Zeeman-split atomic states, which are then detected by a secondary electron multiplier (SEM).

$\left[m_{J}(\theta)= \pm 1\right]$. This operator may be expressed as the difference of the projectors onto even and odd eigenstates of $J(\theta)$ :

$$
\begin{aligned}
M(\theta)= & P_{\text {even }}(\theta)-P_{\text {odd }}(\theta) \\
= & e^{i \theta}\left|g_{-2}\right\rangle\left\langle g_{2}\left|+e^{i \theta / 2}\right| g_{-1}\right\rangle\left\langle g_{1}|+| g_{0}\right\rangle\left\langle g_{0}\left|+e^{-i \theta / 2}\right| g_{1}\right\rangle \\
& \times\left\langle g_{-1}\left|+e^{-i \theta}\right| g_{2}\right\rangle\left\langle g_{-2}\right| .
\end{aligned}
$$

Note that the structure of $M(\theta)$ is entirely equivalent to that of $L(\phi)$ defined in Eq. (18), with the polarization-flip operators $L_{ \pm}$replaced by operators $\left|g_{-m}\right\rangle\left\langle g_{m}\right|$ changing the sign of a Zeeman substate. From Eq. (19), the expectation value for triple correlations of the two cavity photons (using the results of Sec. VI) and the atom may be calculated, assuming the system is in state $\left|G H Z^{\prime}\right\rangle \equiv\left(\left|g_{-2}, 2,0\right\rangle+\left|g_{2}, 0,2\right\rangle\right) / \sqrt{2}$,

$$
\left\langle\mathrm{GHZ}^{\prime}\left|L_{1}\left(\phi_{1}\right) L_{2}\left(\phi_{2}\right) M(\theta)\right| \mathrm{GHZ}^{\prime}\right\rangle=\cos \left(\phi_{1}+\phi_{2}+\theta\right) \text {. }
$$

Thus, apart from the different origin of the third angle, Eq. (30) is exactly analogous to Eq. (21). Therefore, the conclusions of Sec. VI apply, in particular the discussion of perfect correlations, which occur when $\phi_{1}+\phi_{2}+\theta$ is an integer multiple of $\pi$.

Results for the actual experimental situation, with photons decaying from the cavity field before the adiabatic passage is complete, must again be obtained by a Monte Carlo wavefunction simulation of the full master equation. In addition to detecting the quantum jumps of the cavity field, the atomic ground state is probed at the end of each trajectory. An outcome for the measurement is chosen randomly, weighted with probabilities obtained from the final wave function, and the result is added to the classical record. Averaged triple correlations obtained as a function of the orientation of the magnetic field vector in the Stern-Gerlach detector are shown in Fig. 23. The cosine function of Eq. (30) is well reproduced, showing that the dynamic generation of GHZ correlations is also effective if the atomic degrees of freedom are entangled with the photons.

From an experimental point of view, GHZ correlations between an atom and two photons may be easier to detect than their three-photon counterpart. With only two photons escaping from the cavity, the fraction of usable detector responses would increase to $50 \%$. In addition, detection of the 


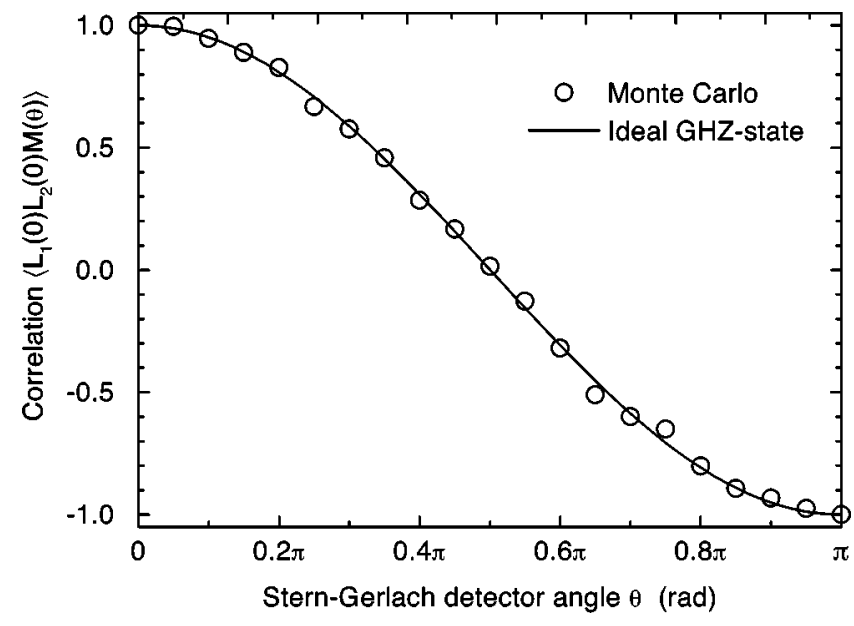

FIG. 23. Monte Carlo wave-function calculation of averaged triple correlations between two cavity decay photons and the atomic state after the adiabatic passage. The parameters are as in Fig. 21 and the cavity decay rate is $\kappa=0.1 \Gamma$. The two photon analyzers are set at an angle $\phi_{1}=\phi_{2}=0$ and only the angle of the magnetic field $\theta$ in the Stern-Gerlach detector is varied. The solid line represents the correlations obtained in the stationary state $\left(\left|g_{-2}, 2,0\right\rangle\right.$ $\left.+\left|g_{2}, 0,2\right\rangle\right) / \sqrt{2}$.

atom provides a trigger signal that can be used to discriminate against background noise in the photon counters. Clearly, another advantage is that there is no need for the preparation of a superposition of Zeeman ground states prior to the adiabatic passage.

\section{CONCLUSIONS}

We have considered a model of adiabatic passage in a strongly coupled atom-cavity system exposed to a classical pump field. With such a setup one may synthesize nonclassical photon states by mapping Zeeman ground-state coherences onto the cavity mode. The original proposal presented in Refs. [20,23] included only a single cavity mode. We have extended that scheme to include two degenerate cavity modes with orthogonal polarization, the case relevant to Fabry-Pérot resonators used in the laboratory. By analytical calculations as well as quantum Monte Carlo simulations we have shown that atomic passage through the system does not lead to pure number states of the two-mode cavity field, as long as atom, cavity, and external pump field are resonant with each other. We could identify diabatic transitions to degenerate dressed levels with higher photon number as the source of this effect.

A central result of the paper is that the adiabatic passage scheme can be modified to work even in the two-polarization case by introducing a finite cavity detuning. This way diabatic transitions are suppressed and states with a definite photon number of the cavity field may be efficiently generated. Several applications of the method were discussed. As a first test of our scheme, we calculated the synthesis of a three photon Fock state of a single polarization of the cavity field and obtained an efficiency above $99 \%$ based on parameters actually achieved in cavity-QED experiments.
The system under study offers intriguing possibilities beyond the generation of number states and their superposition for a single mode. An important property of the atomic transition scheme we employ is its symmetry under a change of sign of (circular) polarization and magnetic quantum number. Taking advantage of this feature allows one to create polarization entangled states of the cavity field. As a particularly relevant example we presented a proposal for the synthesis of a so-called GHZ state consisting of three photons maximally entangled in polarization. It can be used to test EPR correlations without having to resort to Bell-type inequalities.

To probe correlations between the photons generated by our scheme, we make use of the inevitable presence of cavity dissipation. The decay photons emitted from the cavity are sent to linear polarization analyzers of variable orientation. For certain combinations of detector orientations, perfect correlations between the photons may be observed. Most remarkably, these correlations persist even if cavity decay and state synthesis occur on the same time scale.

One should note a difference between the states synthesized in the cavity and the states discussed by GHZ [3]. In the latter case, each particle occupies a distinct mode, while in our cavity the photons are, in principle, not distinguishable. Nonetheless, as demonstrated in Figs. 17 and 23, the observed correlations for a particular subensemble would be precisely as for the "ideal" GHZ state. Qualitatively, this correspondence is due to the temporal evolution of the adiabatic passage and the stochastic nature of cavity decay, with photons usually emerging from the resonator "one by one." This effect could be enhanced through manipulation of the adiabatic passage process: combining suitable shifts of the atomic states with multipeaked interaction profiles $g(t)$ and $\Omega(t)$, the adiabatic passage would proceed in well-separated stages with exactly one photon emitted per stage. With the photons arriving at the detectors at distinct (predetermined) times, the observed correlations generated by the adiabatic passage should correspond exactly to those predicted by GHZ without the need for postselection of a particular subensemble.

A practical concern for the current scheme is the limited time resolution of the detectors, which may not be sufficient to separate the decay events. In this case one might simply register only events in which each of the three photons is actually detected by a separate detector. Note that, in the three particle interferometer orginally proposed by GHZ [4], the correct direction and energy of the particles must also be enforced by apertures around the source and filters in front of the detectors.

We did not discuss the influence of finite detection efficiency on the correlations observed. However, as long as the number of photons generated does not exceed three (a condition that, as we have demonstrated, may be fulfilled by chosing a sufficiently large cavity detuning or coherent coupling $g_{0}$ ), missing a photon decaying from the cavity would only lower the coincidence rate observed, but would not lead to modified three photon correlations. The reason is that events with less than three detected photons can easily be discarded in a coincidence measurement. This argument is 
sometimes called the fair sampling assumption [40].

The proposed method for the synthesis of entangled states is not restricted to the specific cases discussed in the paper. More complicated entangled states of the cavity field may be generated as well. Examples include superposition states with different photon number in the two polarization modes (e.g., $|3,0\rangle+|0,2\rangle$ ) or entangled states with polarization distributed in a more general way (e.g., $|4,2\rangle+|1,3\rangle$ ), as would be useful in quantum information networks with error correction [26].

Maximally entangled states with more than three particles, as discussed by Mermin [50], are of particular interest, since they provide an exponentially stronger test of local realism. These states may be generated with our scheme without any modification, if an atomic transition with the corresponding angular momentum quantum number is used. In cesium, a four particle generalization of the GHZ state may be synthesized with the $F_{g}=4 \rightarrow F_{e}=4$ hyperfine component of the D2 line. However, with increasing number of particles, detection of the correlations between them becomes considerably more challenging, since the coincidence rate would be severely reduced.

The possibilities for the generation of entanglement in our system are further expanded by including atom-photon correlations in the considerations. A corresponding experiment would offer at least two additional benefits: instead of a coherent superposition of Zeeman sublevels, only a single atomic substate is needed as an initial condition for the adiabatic passage, so that the preparation of the atomic state would require only standard optical pumping. Furthermore, detection of the atom would provide a gate for the photon detectors, increasing the signal to noise ratio.

In this paper we have restricted our attention to the case of a dilute atomic beam passing through a cavity. In view of experimental progress in the field [32,33], one may also consider atoms moving through the cavity at a low enough speed to be considered stationary on the timescale set by the coherent interaction, or even trapped atoms or ions in a cavity. The method presented here could also be applied to the case of stationary atoms in a cavity, if the dynamics that in ordinary adiabatic passage is induced by the atom's motion, is generated instead by suitably timed pulses. The interaction with the two cavity modes may be controlled by coupling the atomic levels with a Raman transition using the cavity field and an auxiliary classical field. As suggested in Refs. $[22,25,26]$, the Raman coupling strength may then be adjusted by changing the intensity of the auxiliary field.

\section{ACKNOWLEDGMENTS}

The authors wish to thank Hideo Mabuchi, Sze Men Tan, and Peter Zoller for valuable discussions, and S.M.T. especially for his assistance with setting up the Monte Carlo simulations. This work was supported by the Defense Advanced Projects Agency via the initiative for Quantum Information and Computation (QUIC) administered by the Army Research Office, by the National Science Foundation, and by the Office of Naval Research. W.L. also gratefully acknowledges support from the Deutsche Forschungsgemeinschaft.
[1] J.S. Bell, Physics (Long Island City, N.Y.) 1, 195 (1965).

[2] A. Einstein, B. Podolsky, and N. Rosen, Phys. Rev. 47, 777 (1935).

[3] D.M. Greenberger, M. A. Horne, and A. Zeilinger, in Bell's Theorem, Quantum Theory and Conceptions of the Universe, edited by M. Kafatos (Kluwer Academic, Dordrecht, 1989), pp. 69-72.

[4] D.M. Greenberger, M.A. Horne, A. Shimony, and A. Zeilinger, Am. J. Phys. 58, 1131 (1990).

[5] N.D. Mermin, Am. J. Phys. 58, 731 (1990).

[6] M. Zukowski, Phys. Lett. A 157, 203 (1991).

[7] M.D. Reid and W.J. Munro, Phys. Rev. Lett. 69, 997 (1992).

[8] D.N. Klyshko, Phys. Lett. A 172, 399 (1993).

[9] J.I. Cirac and P. Zoller, Phys. Rev. A 50, R2799 (1994).

[10] S. Haroche, Ann. (N.Y.) Acad. Sci. 755, 73 (1995).

[11] C.C. Gerry, Phys. Rev. A 53, 4591 (1996).

[12] C.C. Gerry, Phys. Rev. A 53, 2857 (1996).

[13] C.C. Gerry, Phys. Rev. A 54, R2529 (1996).

[14] L. Hardy, Phys. Lett. A 160, 1 (1991).

[15] K. Wodkiewicz, L. Wang, and J.H. Eberly, Phys. Rev. A 47, 3280 (1993).

[16] B. Yurke and D. Stoler, Phys. Rev. Lett. 68, 1251 (1992).

[17] A. Zeilinger, M.A. Horne, H. Weinfurter, and M. Zukowski, Phys. Rev. Lett. 78, 3031 (1997).

[18] D. Bouwmeester, J.W. Pan, M. Daniell, H. Weinfurter, and A.
Zeilinger, Phys. Rev. Lett. 82, 1345 (1999).

[19] D.J. Wineland, J.J. Bollinger, W.M. Itano, F.L. Moore, and D.J. Heinzen, Phys. Rev. A 46, R6797 (1992).

[20] A.S. Parkins, P. Marte, P. Zoller, and H.J. Kimble, Phys. Rev. Lett. 71, 3095 (1993).

[21] K. Vogel, V.M. Akulin, and W.P. Schleich, Phys. Rev. Lett. 71, 1816 (1993).

[22] C.K. Law and J.H. Eberly, Phys. Rev. Lett. 76, 1055 (1996).

[23] A.S. Parkins, P. Marte, P. Zoller, O. Carnal, and H.J. Kimble, Phys. Rev. A 51, 1578 (1995).

[24] A. Kuhn, M. Hennrich, T. Bondo, and G. Rempe, Appl. Phys. B: Lasers Opt. 69, 373 (1999).

[25] C.K. Law and H.J. Kimble, J. Mod. Opt. 44, 2067 (1997).

[26] J.I. Cirac, P. Zoller, H.J. Kimble, and H. Mabuchi, Phys. Rev. Lett. 78, 3221 (1997).

[27] Z. Carter, J.R. Gardner, and D.J. Heinzen, Bull. Am. Phys. Soc. 40, 1330 (1995).

[28] C.K. Law and J.H. Eberly, Opt. Express 2, 368 (1998). The method presented in this paper is easily adapted to generate a superposition of the two outer Zeeman states. Initially the ( $F$ $\left.=3, m_{F}=-1\right)$ state is prepared, for example, by optical pumping into $\left(F=3, m_{F}=0\right)$ followed by a Raman $\pi$ pulse using polarizations such that $\Delta m_{F}=-1$. In the second step a coherent superposition of the states $\left(F=3, m_{F}=-1\right)$ and ( $F$ $\left.=4, m_{F}=-1\right)$ is created by application of a Raman $\pi / 2$ pulse 
between the two ground-state hyperfine components $F=3$ and $F=4$. The desired final state is reached by a series of five Raman $\pi$ pulses. The polarizations are chosen such that in the Raman transitions from $F=3$ to $F=4$ the magnetic quantum numbers change in the sequence $\Delta m_{F}=-1,+1,-1,0,-1$.

[29] H.J. Kimble, O. Carnal, N. Georgiades, H. Mabuchi, E.S. Polzik, R.J. Thompson, and Q.A. Turchette, in Atomic Physics 14, edited by D.J. Wineland et al., AIP Conf. Proc. No. 323 (AIP, Woodbury, NY, 1995), pp. 314-35.

[30] Q.A. Turchette, R.J. Thompson, and H.J. Kimble, Appl. Phys. B: Lasers Opt. 60, S1 (1995).

[31] Q.A. Turchette, C.J. Hood, W. Lange, H. Mabuchi, and H.J. Kimble, Phys. Rev. Lett. 75, 4710 (1995).

[32] H. Mabuchi, Q.A. Turchette, M.S. Chapman, and H.J. Kimble, Opt. Lett. 21, 1393 (1996).

[33] C.J. Hood, M.S. Chapman, T.W. Lynn, and H.J. Kimble, Phys. Rev. Lett. 80, 4157 (1998). The parameters for this system are $\left(g_{0}, \Gamma, \kappa\right) / 2 \pi=(120,5.2,40) \mathrm{MHz}$. Using the ultralow loss mirrors of Ref. [44] would result in a tenfold increase of the cavity decay time. Even larger photon storage times of up to $50 \mu$ s are possible by employing whispering gallery modes of $15-\mu \mathrm{m}$ fused-silica microspheres $[51,45,46]$.

[34] J. Oreg, F.T. Hioe, and J.H. Eberly, Phys. Rev. A 29, 690 (1984).

[35] R. Dum, P. Zoller, and H. Ritsch, Phys. Rev. A 45, 4879 (1992).

[36] K. Mølmer and Y. Castin, J. Opt. Soc. Am. B 10, 524 (1993).

[37] H.J. Carmichael, in An Open Systems Approach to Quantum
Optics, Vol. 18 of Lecture Notes in Physics, edited by W. Beiglböck (Springer, New York, 1993).

[38] R. Dum, A.S. Parkins, P. Zoller, and C.W. Gardiner, Phys. Rev. A 46, 4382 (1992).

[39] H. Mabuchi and P. Zoller, Phys. Rev. Lett. 76, 3108 (1995).

[40] J.F. Clauser, M.A. Horne, A. Shimony, and R.A. Holt, Phys. Rev. Lett. 23, 880 (1969).

[41] For a review see J.F. Clauser and A. Shimony, Rep. Prog. Phys. 41, 1881 (1978).

[42] A.S. Parkins (private communication).

[43] R. Walser, J.I. Cirac, and P. Zoller, Phys. Rev. Lett. 77, 2658 (1996).

[44] G. Rempe, R.J. Thompson, H.J. Kimble, and R. Lalezari, Opt. Lett. 17, 363 (1992).

[45] H. Mabuchi and H.J. Kimble, Opt. Lett. 19, 749 (1994).

[46] M. Gorodetsky, A. Savchenkov, and V. Ilchenko, Opt. Lett. 21, 453 (1995).

[47] L. Collot, V. Lefevre-Seguin, M. Brune, J.M. Raimond, and S. Haroche, Europhys. Lett. 23, 327 (1993).

[48] A. Faulstich, A. Schnetz, M. Sigel, T. Sleator, O. Carnal, V. Balykin, H. Takuma, and J. Mlynek, Europhys. Lett. 17, 393 (1992).

[49] M.K. Oberthaler, R. Abfalterer, S. Bernet, J. Schmiedmayer, and A. Zeilinger, Phys. Rev. Lett. 77, 4980 (1996).

[50] N.D. Mermin, Phys. Rev. Lett. 65, 1838 (1990).

[51] V.B. Braginsky, M.L. Gorodetsky, and V.S. Ilchenko, Phys. Lett. A 137, 393 (1989). 